\title{
Europäisches Regieren in der Kartellkontrolle - Probleme und Perspektiven politischer Steuerung durch hierarchisierte transgouvernementale Netzwerke ${ }^{1}$
}

\section{Kurzfassung}

Mit der Dezentralisierung ihrer Kartellpolitik im Jahre 2004 hat sich die EU einen neuen Modus europäischen Regierens gegeben. Sie ersetzt mit dem European Competition Network (ECN), also der Netzwerkbildung der Kartellbehörden der 27 Mitgliedstaaten und der Kommission, einen hierarchischen Steuerungsmodus durch ein transgouvernementales hierarchisiertes Netzwerk. Dieses ist nicht widerspruchsfrei, weil sich unter der Oberfläche formaler Netzwerkstrukturen durch Netzwerkasymmetrien und Autonomiechancen der Netzwerkteilnehmer begründete Fliehkräfte bemerkbar machen. Damit wirft diese Fallanalyse ein neues Licht auf die bisherigen Annahmen zur Funktionsweise transgouvernementaler Netzwerke.

1 Dieser Beitrag entstand im Rahmen des von der Deutschen Forschungsgemeinschaft geförderten Projekts „Netzwerke in der Kartellkontrolle. Ein Dreiländervergleich Deutschland, UK, Ungarn“. Die Autoren danken der DFG für die Unterstützung ihrer Forschungsarbeit. Dank geht auch an drei anonyme Gutachter, die wertvolle Hinweise zur Überarbeitung des Artikels gegeben haben. 


\section{Inhalt}

1. Europäisches Regieren und politische Steuerung durch Netzwerke

2. Die Reform der europäischen Kartellkontrolle: Von der Hierarchie zum Netzwerk

3. Das ECN als integriertes transgouvernementales Netzwerk

a) Aufgaben

b) Funktionsweise

c) Effektivität

4. Transgouvernementales Regieren in der Kartellkontrolle - Schritte zur Enthierarchisierung eines transgouvernementalen Netzwerks?

\section{Europäisches Regieren und politische Steuerung durch Netzwerke}

Das Thema „Europäisches Regieren“ wurde nicht zuletzt deshalb zu einem wichtigen Gegenstand politikwissenschaftlicher Forschung, weil sich hier neue, d. h. auf nationalstaatlicher Ebene nicht zu beobachtende Formen von Staatlichkeit und Staatshandeln untersuchen ließen (Kohler-Koch 1999: 14 f.; Börzel 2006b). Ein wichtiger Aspekt ist hierbei die im europäischen Kontext notwendige Segmentierung der Untersuchung von Staatshandeln, die über das Eigengewicht einzelner Politikfelder hinaus mit der Logik der Europäischen Verträge zu begründen ist. Da die Europäisierung von Politikfeldern vertraglich auf dem Prinzip der Einzelermächtigung beruht, kann jedes Politikfeld mit einem eigenen Entscheidungsmodus ausgestattet werden. Aber auch nachdem die Kompetenzen der europäischen Institutionen klar geregelt sind, bleibt der Kommission ein erheblicher Spielraum bei der Umsetzung der Verträge, den diese auch wahrnimmt (Knill/Lenschow 2003: 1).

Hatte sich die Union in ihrer Regierungstätigkeit anfangs vor allem auf die sogenannte Gemeinschaftsmethode einer rein regulativen Politik mit Hilfe von Verordnungen und Richtlinien gestützt (Eberlein/Kerwer 2002: 2), wandte sie sich mit der zunehmenden Ausdifferenzierung ihrer Aufgaben und der wachsenden Komplexität ihres Regierungshandelns neuen Formen des Regierens zu. Die EU-Forschung hat frühere Unterscheidungen der Organisationsforschung (Powell 1990) aufgegriffen und idealtypisch drei Medien politischer Steuerung unterschieden: Hierarchie, Netzwerke und Markt (Benz 2007: 35). Mit Hierarchie wird ein Steuerungsmodus bezeichnet, der das Handeln dem Politikfeld zugehöriger Akteure per „hoheitlicher“ Weisung koordiniert und 
der die Möglichkeit beinhaltet, Zwang auszuüben und abweichendes Verhalten zu sanktionieren (Börzel 2006b: 76). Die nichthierarchischen Steuerungsformen implizieren einen eher indirekten Ansatz zum Erreichen von Verhaltensänderungen der im Politikfeld relevanten Akteure (Knill/Lenschow 2003: 2). Sie setzen auf Mechanismen wie gegenseitiges Lernen, Überzeugung, Standardisierung von Wissen, Zeitmanagement und Selbststeuerung (Schmidt/ Blauberger/van den Nouland 2007: 273; Héritier 2002a: 3). Die Kommission wird dabei quasi in der Rolle eines ,politischen Unternehmers“ gesehen, der sein europäisches Drehbuch schreibt, aber nationalen politischen Akteuren in der Umsetzung weitgehend die Bühne überlässt (Kohler-Koch 1999: 18). Um das Dilemma zu lösen, dass gemeinsame Standards durch losen Austausch zwar entstehen können, die Durchsetzung jedoch ungewiss bleibt, bietet sich die Beteiligung der im jeweiligen Politikfeld relevanten "Spieler" in Netzwerken an. Dabei stehen sich entweder öffentliche Akteure unterschiedlicher staatlicher Ebenen oder private und öffentliche Akteure gleichberechtigt gegenüber. Diese sind aber mit unterschiedlichen Handlungsressourcen (z. B. Wissen, Geld, Macht, Recht) auch innerhalb der jeweiligen Gruppe (privat/öffentlich) ausgestattet. Ein solches Netzwerk soll im Folgenden näher betrachtet werden. In Abgrenzung zu Netzwerken verfügen Märkte am anderen Ende der „Steuerungsskala" nicht über eine strukturelle Koppelung der Akteure. Stattdessen gestalten die Akteure ihre Handlungen völlig autonom (Börzel 2006b: 76; 2007: 61-63; Tömmel 2007: 16).

Für die Kommission stellt sich bei der Implementation europäischer Politiken, die weder durch Hierarchie noch durch die Selbststeuerung von Märkten kontrolliert werden, immer die gleiche Frage: Wie kann mit den begrenzten Möglichkeiten der Kommission das von ihr gewünschte Ziel im Zusammenspiel mit den Mitgliedstaaten und deren relevanten Institutionen sowie den zahlreichen nichtstaatlichen Akteuren (einschließlich zivilgesellschaftlicher Adressaten) erreicht werden? Diese Frage lässt sich auf zwei Ebenen beantworten. Einerseits wird hier ein Prinzipal-Agent-Problem thematisiert, mit der EU-Kommission in der Rolle des Prinzipals. Andererseits ist die Aufzählung der policy-relevanten Akteure zunächst deskriptiv (Rhodes 2008: 426; Dowding 1995: 137) bzw. als „Metapher“ (Marsh/Smith 2001: 535) mit dem zuvor angerissenen Begriff des „Netzwerks“ zu übersetzen. Rhodes (2008) folgend lässt sich in diesem Kontext ein dreifacher Gebrauch der Netzwerk-Metapher unterscheiden. Erstens als nichthierarchisiertes Interessengeflecht im zuvor geschilderten Sinn, das zu pluralistischem Entscheiden fähig ist, eine Beobachtung, die im europäischen Kontext eher bei der Anwendung der offenen Metho- 
de der Koordination zu beobachten sein wird, beispielsweise in der Sozialpolitik. ${ }^{2}$ Zweitens als interorganisatorisches Entscheidungsmodell. Hier könnte auf europäischer Ebene beispielsweise das Funktionieren des Europäischen Systems der Zentralbanken untersucht werden. Und drittens schließlich als ein bestimmtes Modell europäischen Regierens (governance). Rhodes (2008: 429) stellt dazu fest: „The roots of policy network analysis lie, finally, in the analysis of the sharing of power between public and private actors, most commonly between business, trade unions and the government in economic policy-making." Diese Reduzierung des governance-Begriffes quasi auf die Inhalte der alten Korporatismusdebatte ist sicherlich kritisch zu betrachten. Rhodes selbst hat an anderer Stelle (Rhodes 2000: 61) deutlich gemacht, dass für ihn, neben der Selbstorganisation der Akteure, der auf Vertrauen basierende regelgebundene Austausch auch in Verhandlungsprozessen eine wichtige Rolle spielt. Neuerdings wurde zudem darauf hingewiesen, dass bei diesem dritten Verständnis des Netzwerk-Begriffs eine analytische Unterscheidung zwischen dem Regieren mit Netzwerken und dem Regieren durch Netzwerke erforderlich ist, wobei ersteres sich durchaus auch als hierarchisiertes Regieren vorstellen lässt (Damgard 2006).

Im Folgenden geht es um europäische Kartellpolitik. Festzuhalten ist zunächst, dass das Netzwerk europäischer Kartellbehörden, das den Mittelpunkt der hier angestellten Analyse darstellt, dem Gebrauch des Netzwerkbegriffs als Modell europäischen Regierens entspricht. Vor diesem Hintergrund sind zwei zentrale Fragen zu klären:

1. Welche Charakteristika weisen die entscheidenden Akteure bzw. Adressaten der steuernden Intervention des Prinzipals auf?

2. Wie sieht der vorherrschende Steuerungsmodus in der Kartellpolitik konkret aus?

Die entscheidenden Akteure sind in erster Linie die nationalen Wettbewerbsbehörden, weshalb zum einen die Machtteilung zwischen privatem und öffentlichen Sektor keine Rolle spielt und es sich zum anderen auch nicht nur um ein transgouvernementales, sondern teilweise auch um ein interorganisatorisches Netzwerk handelt, allerdings mit größeren Handlungsspielräumen als dies idealtypischen interorganisatorischen Netzwerken zu eigen ist. Weitgehend unumstritten ist, dass die Kommission mit der formalen Abgabe von Macht zugunsten des Netzwerks einen innovativen Weg gegangen ist. Ob die

2 Vgl. Falkner u.a. 2005; Schmidt/Blauberger/van den Nouland 2007; Knill/Lenschow 2003 und Eberlein/Kerwer 2004. 
nationalen Wettbewerbsbehörden durch ihre formale Aufwertung im Prozess der Gestaltung europäischer Wettbewerbspolitik und ihre Beteiligung im Netzwerk europäischer Kartellbehörden aber tatsächlich an Macht gewonnen haben, oder diese letztlich weiterhin primär bei der Kommission liegt, das ist auch fünf Jahre nach der Neuausrichtung in der europäischen Kartellkontrolle noch strittig. Kontraintuitiv wird argumentiert, dass die Reformen die Stellung der Kommission noch weiter ausgebaut haben. ${ }^{3}$ Es lohnt sich also, genauer hinzusehen.

Die Frage nach dem vorherrschenden Steuerungsmodus in der Kartellpolitik zu beantworten fällt nicht leicht. Wie die Vergangenheit zeigt, lässt sich europäische Kartellpolitik durchaus hierarchisch steuern, weshalb es nahe liegen würde, dem theoretischen Angebot von Eberlein und Newman (2008) zu folgen und das Modell eines hierarchisierten transgouvernementalen Netzwerks auf das Regieren der EU in der Kartellpolitik zu übertragen. Übersetzt in die Umgebung der Kartellpolitik muss dabei die Frage gestellt werden, ob die „,agents“ (also die nationalen Kartellämter) in der Lage sind, dem „principal“ in Gestalt der Kommission Machtressourcen entgegenzusetzen, die Spielräume schaffen, Eigenständigkeit erlauben und damit die Ergebnisse europäischen Regierens modifizieren. Des weiteren wäre nach dem institutionellen Eigeninteresse der beteiligten nationalen Wettbewerbsbehörden im weitesten Sinne, einschließlich der Verteidigung ihrer historisch gewachsenen Wettbewerbskulturen zu fragen, und nach den Chancen und Folgen, die das entsprechende Nutzen von Handlungsspielräumen bietet.

Abzugrenzen ist das Konzept von Eberlein und Newman in verschiedener Hinsicht zu „herkömmlichen“" politikwissenschaftlichen Netzwerkansätzen, die vor allem die Rolle von nicht-staatlichen Akteuren in den Mittelpunkt stellen und Fragen nach Steuerung und Partizipation von Interessengruppen stellen. Im interorganisationellen Analyseraster von Eberlein und Newman steht die Funktionsfähigkeit von Verwaltungsnetzwerken im Mittelpunkt, und weniger die in der politikwissenschaftlichen Netzwerkdebatte im Zusammenhang mit Governance diskutierte Frage nach neuen Formen des Regierens. Im Übrigen ist zentrale Kategorie in allen Netzwerken und den entsprechenden Theorien das Prinzip der Koordinierung als „heiliger Gral“ (Rhodes 2008: 440).

3 Siehe Riley 2003, Wilks 2005, Budzinski 2006, Gerber 2008: 1264.

4 Es ist bereits an dieser Stelle zu vermuten, dass das Theorieangebot der Analyse hierarchisierter transgouvernementaler Netzwerke für den Fall der europäischen Kartellpolitik im Folgenden u. U. modifiziert werden muss. 
Die vorliegende Analyse weist über den konkreten Forschungsgegenstand hinaus. Die nähere Betrachtung des Netzwerks in der Kartellkontrolle ist schon deswegen von hoher Relevanz, weil die Kommission auf anderen Politikfeldern einen ähnlichen Ansatz verfolgt. Aktuellstes Beispiel ist das neu geschaffene Netzwerk der Finanzaufsichtsbehörden. Im Spannungsfeld zwischen einer Zunahme an Aufgaben und Komplexität auf der einen Seite und knappen Ressourcen auf der anderen Seite erscheint die formale Abgabe von Aufgaben bei gleichzeitiger Sicherung von Kongruenz durch Netzwerkregieren ein logisches Modell. Die folgende Untersuchung soll in dieser Hinsicht dreierlei leisten:

1) Sie soll die Erklärungskraft der bisherigen Forschungsergebnisse zum Regieren in hierarchisierten transgouvernementalen Netzwerken im Hinblick auf deren Plausibilität und eventuelle Erweiterungsmöglichkeiten überprüfen,

2) die Annahmen zur Funktionsweise transgouvernementaler Netzwerke mit bisher noch nicht gehobenen empirischen Material testen und

3) Möglichkeiten aufzeigen, wie die Logik europäischen Regierens zwischen Hierarchie und Autonomie in solchen Netzwerken vor allem bezogen auf die Steuerungsambitionen der Kommission weiter entwickelt werden kann.

Die Datenerhebung ist in diesem Politikfeld mit besonderen Schwierigkeiten verbunden. Zum einen haben die untersuchten Behörden stets die Sorge, es könnten Informationen nach außen dringen, die laufende oder künftige Untersuchungsverfahren gefährden. Zum anderen ist gerade während der Interviews mit den für das ECN zuständigen Abteilungen in exemplarisch ausgewählten Behörden - der Generaldirektion Wettbewerb, dem britischen Office of Fair Trading (OFT) sowie der Competition Commission, dem deutschen Bundeskartellamt und der ungarischen Wettbewerbsbehörde GVH - deutlich geworden, dass sozialwissenschaftliche Fragestellungen den Wettbewerbsrechtsexperten eher fremd sind. Dennoch gingen wichtige Impulse von den insgesamt sieben leitfadengestützten Experteninterviews aus. Der jeweilige Leitfaden war im Rahmen des Grundthemas (Funktionsweise des Netzwerks, Kongruenz der Entscheidungslogiken) bewusst offen gestaltet. Die Auswertung von offiziellen Dokumenten der Kommission und Gerichtsakten war ebenfalls ein wichtiges Erhebungsinstrument. Die generelle Zurückhaltung der Experten hat sich im Übrigen auch in der Rücklaufquote einer schriftlichen Befragung unter den am ECN partizipierenden Wettbewerbsbehörden gezeigt, die wir im Untersuchungszeitraum zwischen 2007 und 2008 durchgeführt haben. Gefragt wurde u. a. nach der Steuerung des Netzwerks sowie den Entscheidungs- und Handlungsmaximen der Wettbewerbsbehörden. Immerhin ist 
es gelungen, aus 14 Ländern ausgefüllte Fragebögen zurückzubekommen. Im statistischen Sinne repräsentative Aussagen sind auf diese Weise nicht möglich; dennoch liefern die Befragungsdaten erste Hinweise und Indizien hinsichtlich der Funktionsweise und Logiken, die das Netzwerk der europäischen Kartellkontrolle beherrschen. Diese bieten mögliche Ansatzpunkte für weitere Forschung zum Thema.

Mit der Wettbewerbspolitik wird ein Politikfeld zur Grundlage der Analyse gemacht, das von der EU-Policy-Forschung bisher zu Unrecht wenig beachtet wurde. Die Wettbewerbspolitik steht ,,an der Wiege“ der Römischen Verträge und ist Kern des Binnenmarktprojekts. Sie ist: „EU‘s first truly supranational policy“ (Cini/McGowan 1998: 19). Der EG-Vertrag hatte den ausdrücklichen Schutz des Wettbewerbs nach Artikel 3 Absatz 1 EGV als selbstständiges Ziel der EU vorgesehen. Lange war insofern schon aus vertragsrechtlicher Sicht klar, dass ohne den wettbewerblich organisierten Binnenmarkt der politische Erfolg und die politische Legitimation der Europäischen Union unmittelbar infrage gestellt wären. Wettbewerbspolitik wird von der Kommission zudem als wesentliches Element gesehen, um die europäische Wirtschaft zu stärken und damit die Ziele der Lissabon-Strategie bzw. deren Nachfolge-Strategie „Europa 2020“ zu erreichen (Europäische Kommission 2004a: 2). Der Vertrag über die Europäische Union sieht in Artikel 3 Absatz 3 als Ziel der Union ,eine in hohem Maße wettbewerbsfähige soziale Marktwirtschaft" vor. Allerdings hat der Vertrag von Lissabon den Wettbewerbsschutz nicht als eigenständiges Ziel übernommen. Artikel 3 Absatz 1 AEUV kennt die Wettbewerbsregeln nur als Mittel zum Zweck. Diese Änderung ist auf Druck der französischen Regierung zustande gekommen. Es wurde als Kompromiss ein rechtsverbindliches Protokoll zum EUV und AEUV aufgenommen, das den Wettbewerbsschutz als Ziel und Grundlage legislativer Maßnahmen der Union bekräftigt und betont, dass Wettbewerb als Prozess zu erhalten sei. Inwieweit damit der Wettbewerbsschutz an vertragsrechtlicher Bedeutung verloren hat, ist letztlich eine empirische Frage, die erst in einiger Zeit beantwortet werden kann.

Die Argumentation konzentriert sich im Folgenden auf die Kartellkontrolle, deren Reform auf der Ebene der Generaldirektion Wettbewerb gedanklich und in der organisatorischen Praxis explizit das hier zu erörternde Netzwerkkonzept etablierte. Zuvor soll die neue Qualität der reformierten Kartellpolitik kurz erläutert werden, die durch den Wechsel ihres Steuerungsmodus erst Anlass zu dieser Untersuchung bot. 


\section{Die Reform der europäischen Kartellkontrolle: Von der Hierarchie zum Netzwerk}

Mit dem Übergang von einer zentralisierten Anwendung europäischer Wettbewerbsregeln zu einem stärker dezentral ausgerichteten System des Europäischen Netzwerkes der Kartellbehörden (einschließlich der Kommission) hat die Verordnung (VO) 1/2003 eine „neue“ Form politischer Steuerung etabliert. Dies ist schon deshalb bemerkenswert, als der bisherige Weg der europäischen Integration von einer zunehmend stärkeren Zentralisierung von Kompetenzen bei europäischen Institutionen geprägt war. Dass die Union nun Kompetenzen dezentralisiert und abgibt, für die sie zuvor die alleinige Zuständigkeit besaß, war zumindest aus der Perspektive neofunktionalistischer Ansätze zur Erklärung der europäischen Integration nicht zu erwarten.

Die erste Verordnung in der europäischen Wettbewerbspolitik (VO 17/62) aus dem Jahr 1962 hatte ein stark zentralisiertes Policy-Regime strukturell angelegt. Im Kern sah die Verordnung vor, dass alle den zwischenstaatlichen Handel betreffenden Abkommen zwischen Unternehmen bei der Kommission anzumelden und von ihr zu genehmigen seien. Die alte VO gab der Kommission damit eine außerordentlich große Eingriffsmacht zur Durchsetzung des Wettbewerbs zwischen privaten Firmen und sprach ihr die alleinige Befugnis zu, Ausnahmen vom allgemeinen Kartellverbot nach Artikel 101 Absatz 3 AEUV zu genehmigen. ${ }^{5}$ Damit konnten die Kommission und ihre Generaldirektion Wettbewerb (früher GD IV) die europäische Wettbewerbspolitik nahezu im Alleingang gestalten. Rat und Parlament spielten im politischen Prozess, und erst recht im Alltagsgeschäft der Wettbewerbskontrolle, ohnehin eine eher geringe Rolle. Wettbewerbspolitik war „Commission’s Policy“.

Das galt auch für das Verhältnis zwischen Kommission und Mitgliedstaaten. Artikel 9 (3) der VO 17/62 sah vor, dass die nationalen Wettbewerbsbehörden alle eigenen Untersuchungen zu einem Fall aufgeben mussten, wenn

5 Dieser Beitrag rekurriert auf die mit dem im Dezember 2009 in Kraft getretenen Vertrag von Lissabon neu nummerierten Artikel. Die Wettbewerbsartikel 81 und 82 EGV sind wortgleich als Artikel 101 und 102 AEUV übernommen worden. Dies ist bereits die zweite Neunummerierung; die erste war mit dem Vertrag von Amsterdam im Jahre 1997 (in Kraft seit 1999) umgesetzt worden. Vorher wurden die beiden hier betrachteten Wettbewerbsartikel als Artikel 85 und 86 EGV geführt. Um die Lesbarkeit zu vereinfachen und Übersichtlichkeit zu gewährleisten, ist im Folgenden immer von den derzeit gültigen Artikeln 101 und 102 AEUV die Rede, auch dann, wenn Aussagen getroffen werden, die in die Vergangenheit zurückreichen, in der eine der alten Nummerierungen noch Bestand hatte. 
die Kommission selbst eine Untersuchung nach Artikel 101 oder 102 AEUV initiierte. Nationale Behörden hatten der Kommission nach Artikel 11 der Verordnung 17/62 zudem durch Bereitstellung von Informationen zuzuarbeiten. Die Generaldirektion verfügte zudem über zahlreiche informelle Instrumente (z. B. Verwaltungsschreiben oder Gruppenfreistellungsverordnungen), die ihre Stellung zusätzlich stärkten. Im Beratenden Ausschuss („Advisory Committee"), der sich aus Experten der Mitgliedstaaten zusammensetzt, konnten die nationalen Behörden zwar bedingt Einfluss nehmen, die Kontrolle verblieb letztendlich aber bei der Kommission, zumal die Beratungsvorschläge der Mitgliedsländer nicht veröffentlicht werden durften. Insofern fand sich eine deutliche Machtasymmetrie zwischen der europäischen und der nationalen Ebene.

Das zentralisierte System europäischer Kartellkontrolle nach der VO 17/62 hatte jedoch zu einer Überlastung der ohnehin limitierten Ressourcen der Generaldirektion Wettbewerb geführt. Zum Jahresende 2000 beispielsweise waren 935 Kartellfälle noch unerledigt (Sturm/Zimmermann-Steinhart 2003: 392). Angesichts der bevorstehenden erneuten Erweiterung des Binnenmarktes durch den Beitritt der zehn mittel- und osteuropäischen Staaten, von der eine weiter wachsende Zahl an Kartell- und Fusionsfällen zu erwarten war, führte dies schließlich zu umfassenden Reformüberlegungen bei der Kommission. Damit begegnete sie zugleich der anhaltenden Kritik an Arbeitsweise und Methoden der Generaldirektion. Wichtigen Einfluss nahm hier eine „epistemic community“, der Praktiker aus den nationalen Behörden und Gerichten sowie Firmenvertreter und Anwälte angehören (Wilks 2005: 447; van Waarden/Drahos 2002; Kassim/Wright 2009). In dieser „epistemic community“ war die Unzufriedenheit mit dem alten System mittlerweile groß, Artikel 101 AEUV würde zu weit ausgelegt, die Erkenntnisse der Wirtschaftswissenschaft würden zu wenig berücksichtigt und die Verfahren seien zu starr und bürokratisch, so lautete die Kritik (Steinke 2011). Zudem musste die Kommission eine Reihe von Niederlagen vor den europäischen Gerichtshöfen hinnehmen. Zu Beginn des neuen Jahrtausends legte die GD Wettbewerb daraufhin ein Modernisierungspaket vor, das neben der neuen Kartellverfahren auch eine neue Fusionskontrollverordnung, eine Bekanntmachung über das European Competition Network (Netzwerkbekanntmachung) als Teil der Kartellkontrolle sowie einige weitere Papiere umfasste.

Die neue VO 1/2003, die schließlich nach umfassenden Verhandlungen auf verschiedenen Ebenen (Kassim/Wright 2009) am 1. Mai 2004 in Kraft trat, veränderte das europäische Wettbewerbsrecht in zwei fundamentalen Bereichen. Sie ersetzte das Monopol der Kommission auf die Implementation des 
europäischen Wettbewerbsrechts durch ein dezentrales System. Seit der Reform wenden auch die nationalen Kartellbehörden Artikel 101 und 102 AEUV direkt und vollständig an, wobei sie über ähnliche Kompetenzen verfügen wie die Kommission. Erst wenn der Handel zwischen mehr als drei Mitgliedstaaten von einem Verstoß gegen die Wettbewerbsregeln betroffen ist oder neue rechtliche Fragen europaweit zu klären sind, übernimmt meist die Kommission den Fall. Das System sieht somit eine parallele Zuständigkeit aller nationalen europäischen Wettbewerbsbehörden vor. Die zweite fundamentale Veränderung ist der Übergang von der Ex-ante- zur Ex-post-Kontrolle. Mussten Unternehmen geplante Kooperationen vor der Reform bei der Kommission anmelden und die Vereinbarkeit der Absprachen mit dem europäischen Wettbewerbsrecht überprüfen lassen, sieht die VO 1/2003 nun ein Legalausnahmesystem vor, das die Unternehmen von dieser Pflicht befreit. Firmenkooperationen werden nur noch auf Beschwerden oder auf begründeten Verdacht hin auf ihre Vereinbarkeit mit dem europäischen Wettbewerbsrecht untersucht.

Obwohl das Vorhaben anfangs durchaus mächtige Gegner hatte, zu denen z. B. das Bundeskartellamt gehörte, ist der Kommission eine konsequente Umsetzung gelungen. Die Probleme bei der Kompromissfindung, die sich dann ergeben, wenn Mitgliedstaaten mit unterschiedlichem Verständnis von Wettbewerbspolitik an der Entscheidungsfindung beteiligt sind (Müller/Sturm 2010: 159) konnte die Kommission erstaunlich gut lösen. Drei Faktoren haben hier eine Rolle gespielt: Erstens ist es die Generaldirektion Wettbewerb, die Rechtsakte maßgeblich erarbeitet und vorbereitet. So haben die Generaldirektion Wettbewerb bzw. die Kommission de facto die Kontrolle über den legislativen Prozess. Zweitens enthielt die schließlich verabschiedete Verordnung zumindest nominell mehr Befugnisse für die nationalen Wettbewerbsbehörden, was eine Mehrheit im Rat erleichterte. Drittens hat es die Kommission wohl geschafft, auch inhaltlich zu überzeugen und dabei geschickt argumentiert. Auffällig ist beispielsweise, dass die Kommission häufig betont hat, dass sämtliche Neuerungen den Verbrauchern zugutekommen. Diesem Ziel können sich politische Akteure kaum verweigern. Auf Kritik, die neue Vorgehensweise schaffe mehr Rechtsunsicherheit, reagierte die Kommission mit der Schaffung des Netzes der europäischen Wettbewerbsbehörden (European Competition Network, ECN). Dem ECN gehören die Kartellbehörden der 27 Mitgliedstaaten sowie die Generaldirektion Wettbewerb an.

Von der Dezentralisierung der europäischen Wettbewerbskontrolle versprach sich die Kommission eine Entlastung und hoffte, künftig die eigene Arbeit verstärkt priorisieren zu können und so mehr Ressourcen zur Verfügung 
zu haben, z. B. für die Verfolgung von Hardcore-Kartellen oder für die Untersuchungen ganzer Wirtschaftssektoren bei vermutetem Missbrauch von Marktmacht (Europäische Kommission 2004a: 17). Damit haben sich auch die Formen europäischen Regierens in der Wettbewerbspolitik gewandelt. An die Stelle des zentralistischen, hierarchischen Wettbewerbsregimes der VO 17/62 ist ein dezentraleres System der Wettbewerbskontrolle getreten. Der Wegfall des Anmeldesystems bei Unternehmenskooperationen überträgt den Unternehmen die erste Beurteilung darüber, ob das eigene Verhalten mit den europäischen Wettbewerbsregeln konform ist. Und auch das ECN ist insofern ,,selbstregulierend" gedacht, als die nationalen Kartellbehörden in diesem Rahmen ihre Politik, ihr Vorgehen, die Fallverteilung und auch ihre Entscheidungen abstimmen sollen.

Die Kommission geht nun in der Kartellkontrolle den schwierigen Weg zwischen Autonomie und Hierarchie, wobei die Steuerung durch das neugebildete Netzwerk Ineffizienzen bei der Politikimplementation vermeiden soll. Das Steuerungsdilemma, das im Sinne eines Prinzipal-Agent-Verhältnisses hier deutlich wird, nämlich dass der Prinzipal (GD Wettbewerb) den Zugewinn an Effizienz mit einem Verlust an Kontrolle bezahlt, blieb im Netzwerkkonzept, so wie dieses als Reformprojekt formuliert wurde, zwar ungelöst. Der Prinzipal versicherte sich aber einer zusätzlichen Machtressource, nämlich der in Artikel 11 (6) der VO 1/2003 verbrieften Fähigkeit, nach eigenem Gutdünken alle Kartellfälle, die europäisches Recht betreffen, an sich zu ziehen. Dies schuf einen „Schatten der Hierarchie“ im Sinne Scharpfs (1993: 67-68), der noch einmal das erwähnte Steuerungsdilemma deutlich macht. Während die Autonomie der ,agents“ einen stärker an Vertrauen und geteilter Wettbewerbskultur orientierten Steuerungsansatz nahelegen würde, basiert der „Schatten der Hierarchie" auf dem machtpolitischen Durchgriff, der im Extremfall (d. h. wenn er zur Regel wird) jegliche Selbstbezogenheit des Netzwerks und erst recht im Verfahren entstandene nichtjuristische Kooperationsgewinne, wie gemeinsame Handlungsnormen und -konventionen, zunichte machen kann. Dies lässt auch die naheliegende und in der Begründung der Reform von 2004 durchaus vorzufindende Argumentation zweifelhaft erscheinen, die Reform folge der Logik der Durchsetzung des Subsidiaritätsprinzips in der EU (Schmidt/Schmidt 2006: 199). Was das skizzierte Steuerungsdilemma für die Praxis der europäischen Wettbewerbspolitik bedeutet, soll nach einer eingehenderen Analyse der Qualität des ECN als integriertem transgouvernementalem Netzwerk nach Eberlein und Newman (2008) beurteilt werden. 


\section{Das ECN als integriertes transgouvernementales Netzwerk}

Eberlein und Newman haben, wie eingangs bereits angedeutet, ein Raster zur Analyse transgouvernementaler Netzwerke vorgeschlagen, mit dem im Folgenden die Praxis der europäischen Kartellkontrolle erfasst und die neu entstandenen Steuerungsprobleme des ECN herausgearbeitet werden. Das ECN weist zwei zentrale Charakteristika auf, die Eberlein und Newman (2008: $31 \mathrm{f}$.) in Abgrenzung zu anderen, ,gängigeren“ Netzwerken nennen: Zum einen handelt es sich um ein Netzwerk, dem (mit Ausnahme der Generaldirektion Wettbewerb) nur nationale Behörden angehören, also weder Regierungen noch private Akteure; zum anderen beschränkt sich die Mitgliedschaft auf individueller Ebene somit nicht auf Regierungsvertreter, sondern auf Beamte in Wettbewerbsbehörden. Es sind gerade solche Netzwerke, die in der Forschung bislang unterbelichtet sind. Die folgende Analyse gliedert sich entlang der wichtigsten Elemente des o. g. Rasters: Dies sind (a) die Aufgaben, (b) die Funktionsweise und (c) die Effektivität integrierter transgouvernementaler Netzwerke (Eberlein/Newman 2008: 27).

\section{a) Aufgaben}

Die Aufgaben des ECNs unterstreicht bereits die Einleitung der VO 1/2003. Hier heißt es im ersten Erwägungsgrund, das ECN sei Teil eines Systems, „das gewährleistet, dass der Wettbewerb im Gemeinsamen Markt nicht verfälscht wird (...)“. Ausgangspunkt ist hierbei die Stärkung der Beziehungen der Behörden untereinander durch das neu ins Leben gerufene Netzwerk. „Das Netz“, heißt es in der Netzwerkbekanntmachung, ,ist ein Diskussions- und Kooperationsforum für die Anwendung und Durchsetzung der EG-Wettbewerbspolitik.“ Zudem sei es „die Basis für die Schaffung und Wahrung einer gemeinsamen Wettbewerbskultur in Europa" (Europäische Kommission 2004b: 43). Aufgabe des Netzwerks ist es, die Arbeit zwischen den Kartellbehörden effizient zu verteilen und das europäische Wettbewerbsrecht wirksam und kohärent anzuwenden.

\section{b) Funktionsweise}

Hinsichtlich der Funktionsweise schlagen Eberlein und Newman (2008: 32) für die Klassifizierung eines Netzwerks als integriertes transgouvernementales Netzwerk die Indikatoren „hohes Maß an Delegation“ und „hohes Maß an for- 
malen Befugnissen“ (formal authority) vor. Ersteres bedeutet, dass die Behörden keiner nationalen hierarchischen Befehlskette untergeordnet sind und von außen nicht beeinflusst werden. Letzteres verweist auf die Kompetenzen, mit denen die einzelnen Mitglieder eines Netzwerks ausgestattet sind, und kann Hinweise auf die Stärke einer Behörde - sowie des Netzwerks insgesamt - bei der Erfüllung der anfallenden Aufgaben sein. Je mehr Befugnisse einer Behörde zugeteilt wurden, desto durchsetzungsfähiger ist sie potentiell bei der Erfüllung ihrer Aufgaben und auch gegenüber anderen Mitgliedern des Netzwerks.

Tabelle 1 illustriert die Unabhängigkeit der ECN-Mitglieder. Sämtliche Behörden gelten als in hohem Maße frei von politischer Einflussnahme. Entscheidungen bei der Beurteilung von Wettbewerbsverstößen treffen sie entweder selbst, oder ein entsprechendes Wettbewerbsgericht fällt ein Urteil. Ministerien oder andere politische Institutionen haben bei Kartell- oder Missbrauchsfällen keinerlei Mitspracherecht. Allerdings genießt der Wirtschaftsminister oder die ganze Regierung in acht Mitgliedstaaten das Recht, die Entscheidung einer Behörde bei Fusionen zu überstimmen, wenn dies im öffentlichen Interesse ist. Ein Einschreiten der Politik ist zwar selten, jedoch immer wieder zu beobachten, in Deutschland zuletzt bei der Übernahme von Ruhrgas durch E.on. Grundsätzlich ist aber zu konstatieren, dass die Wettbewerbsbehörden - und damit auch das ECN als Ganzes - einen hohen Grad an formaler Unabhängigkeit aufweisen, auch wenn dies in einigen kleineren Ländern im Einzelfall anders sein mag. In allen Wettbewerbsbehörden ist zudem die Unabhängigkeit von politischen Entscheidungen Teil des institutionellen Selbstverständnisses. 


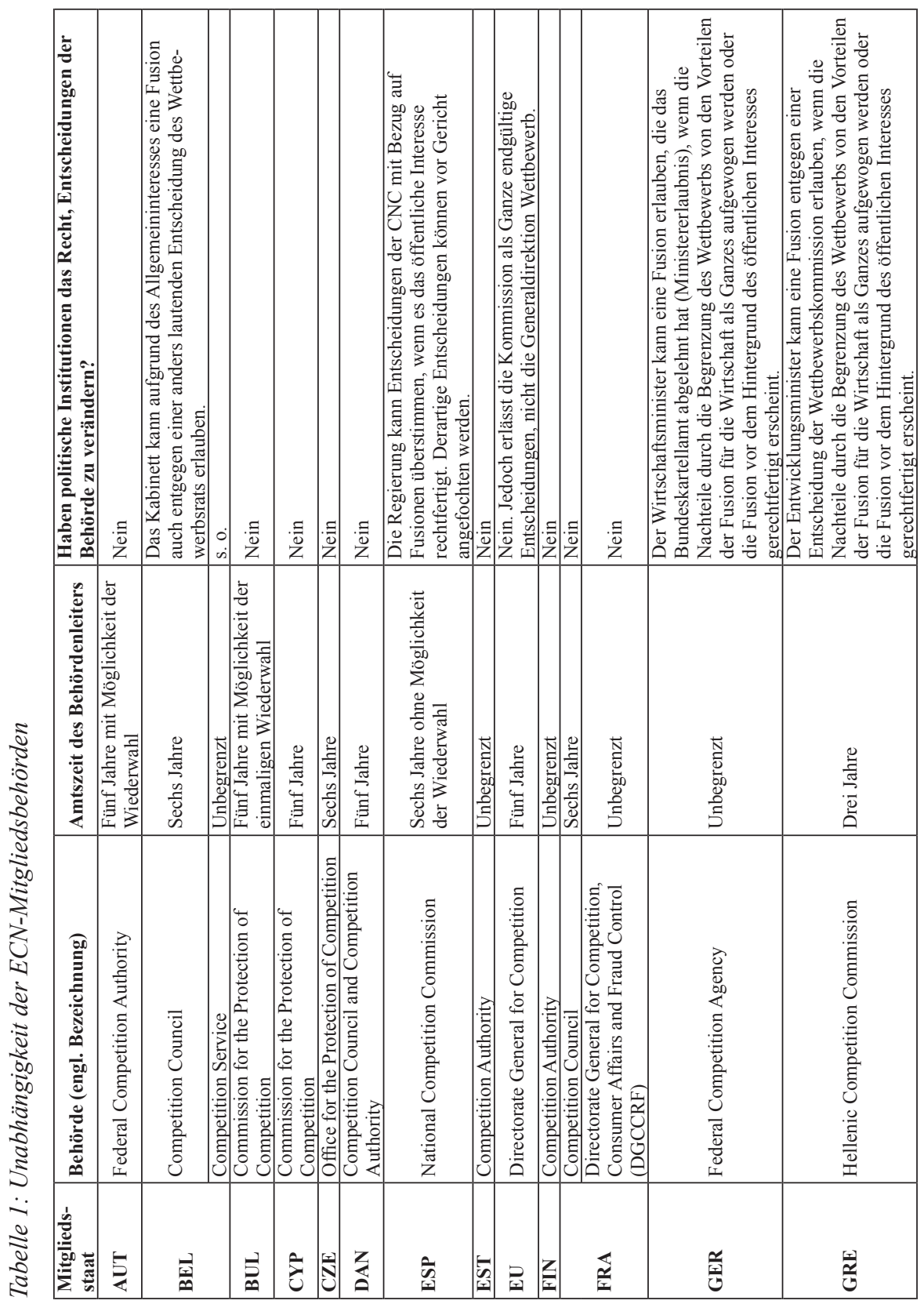




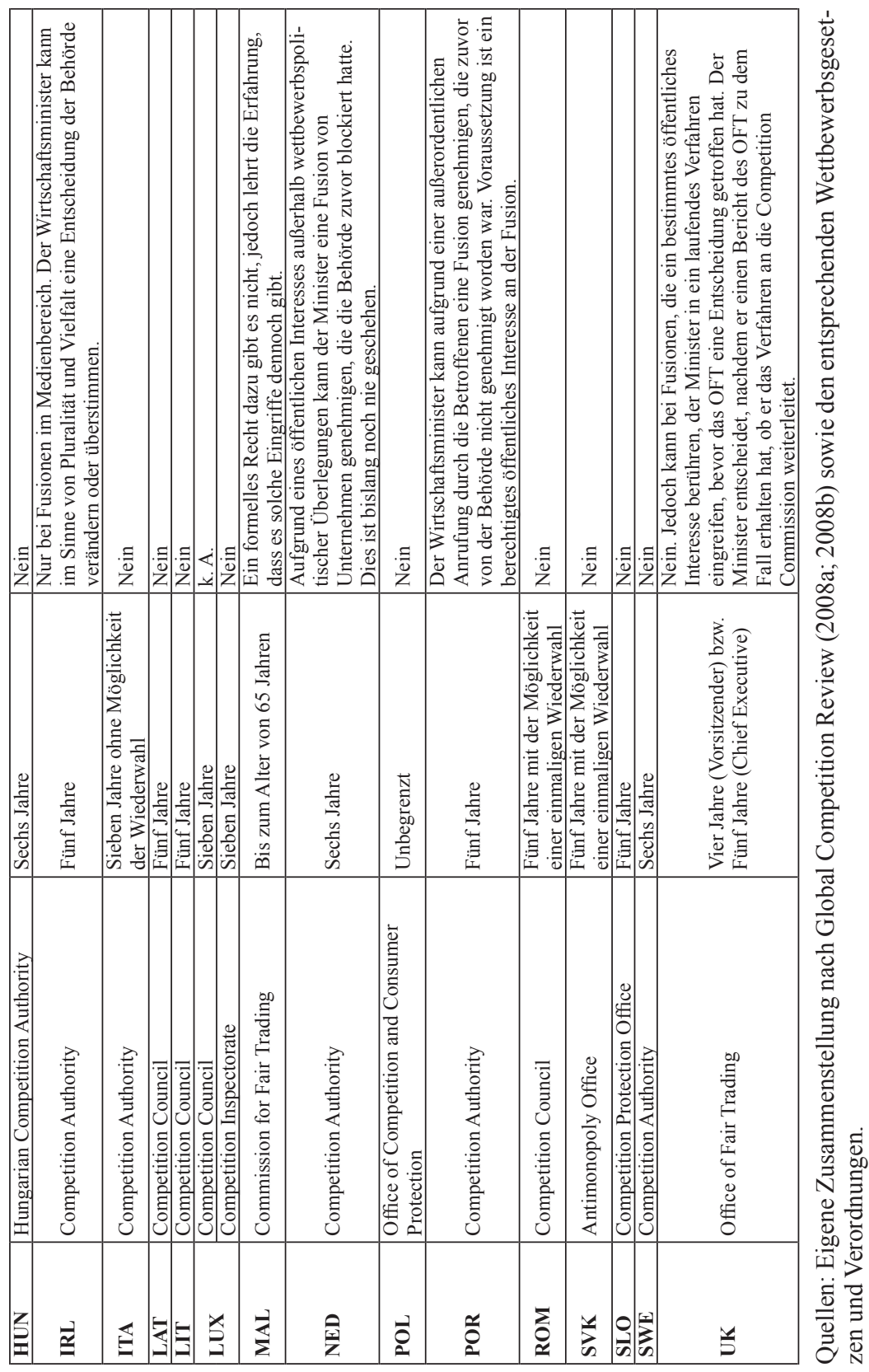


Neben der Unabhängigkeit der Behörden sind auch deren Befugnisse sowie deren Durchsetzungsfähigkeit von Bedeutung. Tabelle 2 zeigt, dass es hinsichtlich ihrer Kompetenzen ein hohes Maß an Konvergenz in den nationalen Wettbewerbsgesetzen gibt. Dies liegt vor allem daran, dass alle EU-Mitgliedstaaten in jüngerer Zeit ihre nationalen Wettbewerbsgesetze an die europäischen Wettbewerbsregeln und die Befugnisse ihrer Behörden an diejenigen der GD Wettbewerb angepasst haben. Zwar bestehen große Unterschiede in der Organisation der Kartellrechtsdurchsetzung - wie bereits erwähnt haben manche Staaten eine Behörde, die zugleich Erstentscheidungsinstanz ist (z. B. Deutschland), andere verfügen über ein zweigliedriges System aus Untersuchungsbehörde und Wettbewerbsgericht (etwa Österreich, Belgien oder Luxemburg). Hinsichtlich der Ermittlungsbefugnisse lässt sich jedoch eine große und wachsende Übereinstimmung feststellen.

Im Allgemeinen ist zu bemerken, dass den Wettbewerbsbehörden grundsätzlich eine starke Stellung zukommt. Alle ermittelnden Behörden haben das Recht, von selbst tätig zu werden (ex officio) und müssen nicht in jedem Fall auf eine Anzeige oder Beschwerden warten. Außerdem sind sie befugt, überraschende Durchsuchungen der verdächtigen Unternehmen (sogenannte dawn raids) durchzuführen. Sie dürfen Beweismaterial sicherstellen, kopieren oder versiegeln, einschließlich elektronischer Beweismittel wie E-Mails oder ganzer Computerfestplatten, und sie haben das Recht, einzelne Personen wie auch Unternehmen zu Auskünften zu zwingen. Bei fehlender Kooperation seitens der Verdächtigen können viele Behörden außerdem Strafen verhängen.

Hinsichtlich der Instrumente der Wettbewerbsbehörden zur Kartellrechtsdurchsetzung lässt sich in der jüngsten Zeit die Tendenz nicht nur zur Vereinheitlichung feststellen, sondern auch ein Trend zur Verschärfung. Darüber hinaus ist eine zunehmende Kriminalisierung von Kartell- und Missbrauchsvergehen zu beobachten. Eine steigende Zahl von Behörden kann bei der Ahndung von Verstößen auch auf das Strafrecht zurückgreifen. Gefängnisstrafen sind möglich. 
Europäisches Regieren in der Kartellkontrolle

\begin{tabular}{|c|c|c|c|c|c|c|c|c|c|c|c|c|c|c|c|}
\hline$\neg \simeq$ & $U \ll$ & $x$ & $x$ & $x$ & $x$ & $x$ & $x$ & ' & . & ' & ' & $x$ & $x$ & $x$ & $x$ \\
\hline エ $\supset$ & $\Xi \cup \ll$ & $x$ & $x$ & $x$ & $x$ & $|x|$ & $x$ & $x$ & $x$ & $x$ & $x$ & $x$ & $x$ & $x$ & $x$ \\
\hline ( & \pm 00 & $x$ & $x$ & $x$ & ' & $x$ & $x$ & $x$ & $x$ & $x$ & $x$ & $x$ & $x$ & $x$ & \\
\hline ك & $\varpi \circlearrowright \ll$ & $x$ & $x$ & $x$ & $x$ & $x$ & $x$ & $x$ & $x$ & $x$ & $x$ & $x$ & $x$ & $x \mid$ & 0 \\
\hline$\omega \simeq \ll$ & I & $x$ & $x$ & $x$ & $x$ & $x$ & $x$ & $x$ & $\Xi$ & $\Xi$ & $x$ & $x$ & $x$ & $x \mid$ & $x$ \\
\hline$\Psi \simeq \ll$ & U U & $x$ & $x$ & $x$ & $x$ & $x \mid$ & $x$ & $x$ & $x$ & $x$ & $x$ & . & . & $x \mid$ & $x$ \\
\hline 山ーZ & 山U & $x$ & $x$ & $x$ & ' & $x$ & $x$ & ' & 1 & ' & $x$ & $x$ & $x$ & $x$ & \\
\hline 凹 & 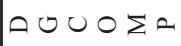 & $x$ & $x$ & $x$ & $x$ & $x$ & $x$ & $x$ & $x$ & $x$ & $x$ & $x$ & $x$ & $x$ & . \\
\hline 山थ & $U \ll$ & $\Xi$ & $\Xi$ & $x$ & ' & $|x|$ & $x$ & $x$ & $x$ & $x$ & ' & $x$ & $x$ & $x:$ & $x$ \\
\hline w $n=$ & $z \cup \cup$ & $x$ & $x$ & $x$ & $x$ & $x \mid$ & $x$ & $x$ & $x \mid$ & $x$ & $x$ & . & . & $x$ & \\
\hline$\theta \ll z$ & $\theta \cup \ll$ & $x$ & $x$ & $x$ & ' & $|x|$ & $x$ & $x$ & $x \mid$ & $x$ & $x$ & $x \mid$ & $x$ & $x$ & $x$ \\
\hline UN & $0 \approx 0$ & $x$ & $x$ & $x$ & $x$ & $x$ & $x$ & $x$ & $x$ & $x$ & $x$ & $x$ & $x$ & $x$ & ' \\
\hline$U>a$ & 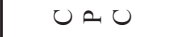 & $x$ & $x$ & $x$ & ' & $|x|$ & $x$ & $x$ & $x \mid$ & $x$ & $x$ & $x$ & $x$ & $x \mid$ & ' \\
\hline ـ D & U U U U & $x$ & $x$ & $x$ & $x$ & $x$ & $x$ & $x$ & $x$ & $x$ & ' & $x$ & $x$ & $x$ & . \\
\hline لـ & U U & $x$ & $x$ & ' & ' & ' & ' & $x$ & $x$ & $x$ & $x$ & $x$ & $x$ & $x$ & . \\
\hline لـ & U & ' & ' & $x$ & $x$ & $|x|$ & $x$ & & . & ' & ' & $x$ & $x$ & $x$ & ' \\
\hline «つ & 山 U & $x$ & $x$ & $x$ & $x$ & $x$ & $x$ & I & . & ' & ' & $x$ & 1 & $x$ & 0 \\
\hline & 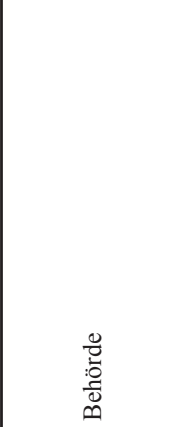 & 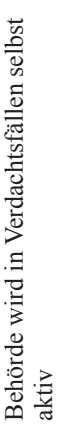 & 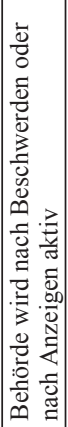 & 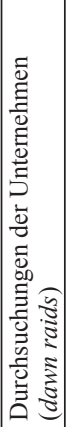 & 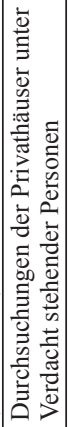 & 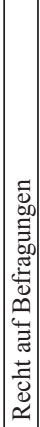 & 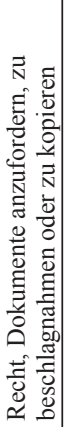 & 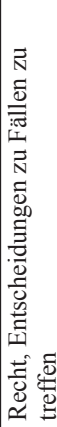 & 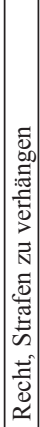 & 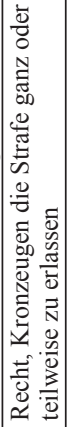 & 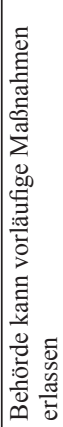 & 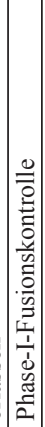 & 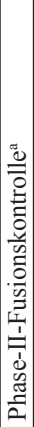 & 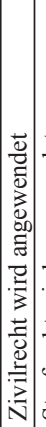 & 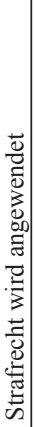 \\
\hline
\end{tabular}

ZPol 3-4/10 


\begin{tabular}{|c|c|c|c|c|c|c|c|c|c|c|c|c|c|c|c|c|}
\hline$\checkmark$ & $O$ w & $x$ & $x$ & $x$ & $x$ & $x$ & $x$ & $x$ & $x$ & $x$ & $x$ & $x$ & & $x$ & $x$ & 氖. \\
\hline$n \geqslant m$ & $n \cup \ll$ & $x$ & $x$ & $x$ & $x$ & $x \mid$ & $x$ & $x$ & 1 & ' & $x$ & $x$ & $x$ & $x$ & ' & \\
\hline$n \rightarrow 0$ & $0 \sim 0$ & $x$ & $x$ & $x$ & $x$ & $x$ & $x$ & 0 & $x$ & & ' & $x$ & $x$ & $x$ & $x$ & \\
\hline$n>v$ & $\ll 0$ & $x$ & $x$ & $x$ & $x$ & $x$ & $x$ & $x$ & $x$ & $x$ & ' & $x$ & $x$ & $x$ & ' & \\
\hline$\simeq O \Sigma$ & U & $x$ & $x$ & $x$ & $x$ & $x \mid$ & $x$ & $x$ & $x \mid$ & $x$ & $x$ & $x$ & $x \mid$ & $|x|$ & $x$ & \\
\hline $0,0 \simeq$ & $0 \ll$ & $x$ & $x$ & $x$ & ' & $x \mid$ & $x$ & $x$ & $x \mid$ & $x$ & $x$ & $x$ & $x$ & $x$ & 1 & \\
\hline • O- & $0 \cup \cup a$ & $x$ & $x$ & $x$ & $x$ & $x$ & $x$ & $x$ & $x$ & $x$ & $x$ & $x$ & $x$ & $x$ & ' & \\
\hline Z曲 D & $U \ll$ & $\Xi$ & $x$ & $x$ & $x$ & $x$ & $x$ & $x$ & $x$ & $x$ & $x$ & $x$ & $x$ & $x$ & ' & \\
\hline$\Sigma \ll\lrcorner$ & U山 & $x$ & $x$ & $x$ & $x$ & $x$ & $x$ & $x$ & $x$ & $x$ & $x$ & $x$ & $x$ & $x$ & ' & \\
\hline • & $\cup \cup$ & $\underset{\longleftarrow}{\longleftarrow}$ & $x$ & ' & ' & ' & ' & $x$ & $x$ & $x$ & $x$ & ' & 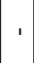 & $x$ & 0 & \\
\hline • つメ & $U-$ & $\Xi$ & $x$ & $x$ & $x$ & $x$ & $x$ & ' & ' & ' & ' & ' & , & $x$ & 0 & \\
\hline •ーヒ & U & $x$ & $x$ & $x$ & ' & $x$ & $x$ & $x$ & $x \mid$ & $x$ & ' & $x$ & $x$ & $x$ & ' & \\
\hline$\lrcorner \ll \models$ & U U & $x$ & $x$ & $x$ & $x$ & $x \mid$ & $x$ & $x$ & $x$ & $x$ & $x$ & $x$ & $x$ & $x$ & & \\
\hline ートく & $\neg U \ll$ & $x$ & $x$ & $x$ & ' & $|x|$ & $x$ & $x$ & $x$ & $x$ & $x$ & $x$ & $x$ & $x$ & ' & \\
\hline & $\begin{array}{l}\overbrace{0}^{0} \\
: 0 \\
0 \\
\infty\end{array}$ & 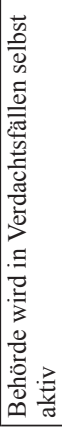 & 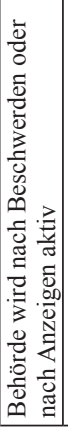 & 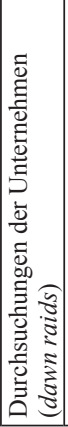 & 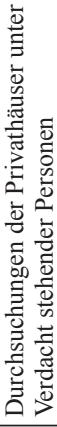 & 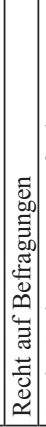 & 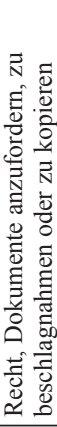 & 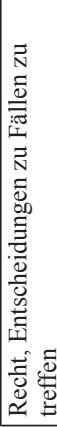 & 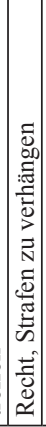 & 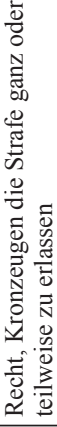 & 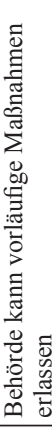 & 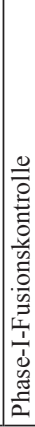 & 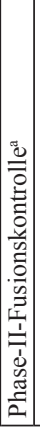 & 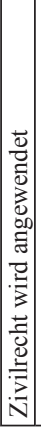 & 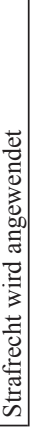 & 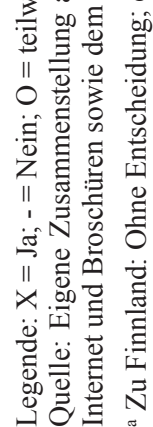 \\
\hline
\end{tabular}


Neben der institutionellen Unabhängigkeit im nationalen Kontext lässt sich eine breite Palette von Instrumenten zur Durchsetzung der nationalen wie der europäischen Wettbewerbsregeln nachweisen, über die die Mitglieder des ECN verfügen. Damit ist gewährleistet, dass diese im Netzwerk autonom handeln können und bei ihrer Zusammenarbeit, auch ohne Einbeziehung der Kommission, nicht an formale Hürden der Kooperation stoßen. Es ist jedoch nicht auszuschließen, dass nationale Wettbewerbsinstitutionen ein ausgeprägtes institutionelles Eigeninteresse am Ausbau ihrer Macht- und Ressourcenbasis haben und deshalb diese Zusammenarbeit eher strategisch als im Sinne einer Stärkung des Netzwerks sehen. Diese Annahme ließe sich theoretisch mit Rational-Choice-Annahmen unterlegen, die in der Bürokratietheorie üblich sind (Niskanen 1971; Peters 2010: 307-310).

In Erweiterung des von Eberlein und Newman vorgeschlagenen Untersuchungsrahmens sollen im Folgenden zusätzlich die Art und die Inhalte von Interaktionen innerhalb des Netzwerks untersucht werden. Dieser Aspekt findet bei Eberlein und Newman eher am Rande Beachtung, hat jedoch über die formalen Befugnisse der Wettbewerbsbehörden hinausreichende und damit im Kern politikwissenschaftliche Relevanz. Die netzwerkinternen Austauschprozesse beeinflussen maßgeblich die Politikergebnisse und -wirkungen. Ihre Steuerung dieser Interaktionen demonstriert in der Praxis wie der Prinzipal (GD Wettbewerb) mit den zur autonomen Strategiefindung fähigen nationalen Wettbewerbsbehörden umgeht, um seine Steuerungsziele zu erreichen. Letztlich stellt sich damit die Machtfrage, da diese die Interaktionen beeinflusst und den Grad der Selbststeuerung des Netzwerks bestimmt.

Das ECN dient als Plattform sowohl der Beschlussfassung in konkreten Fällen als auch der Diskussion über fallbezogene und generelle Fragen der Wettbewerbspolitik. Hinsichtlich der Beschlussfassung sind zwei gemeinsame Intranetplattformen von zentraler Bedeutung, auf die alle Wettbewerbshüter zurückgreifen können. Hier stellen sie relevante und zum Teil auch vertrauliche Informationen ein. So ist es beispielsweise mit Hilfe der Intranetplattform „ECN-Interactive“ möglich, dass sich Mitarbeiter einer Wettbewerbsbehörde genau über die Vorgänge einer anderen Behörde zu einem ähnlichen Fall informieren können. Sind mehrere Länder in einen Fall involviert, sollen die beteiligten Wettbewerbshüter im ECN gemeinsam über die Fall- bzw. Arbeitsverteilung beraten. Das Bundeskartellamt gibt beispielsweise an, in den Jahren 2005 und 2006 zwei Fälle an die Kommission abgegeben zu haben. Enge Abstim- 
mungen mit anderen nationalen Wettbewerbsbehörden, die ähnliche Fälle verfolgten, seien zudem die Regel. ${ }^{6}$

Darüber hinaus bestehen im ECN Arbeitsgruppen, in denen sich Fachleute mit Spezialthemen auseinandersetzen. Neben der Arbeitsgruppe der behördlichen Chefökonomen, die als Austauschforum über den Forschungsstand der wettbewerbsökonomischen Analyse dienen soll, gibt es eine Arbeitsgruppe, die sich mit der Reform der Anwendung des Artikels 102 AEUV beschäftigt. Außerdem gibt es drei horizontal, d. h. sektorübergreifend ausgerichtete Arbeitsgruppen, in denen sich vor allem Mitglieder der mittleren Leitungsebene der Behörden treffen: Die Arbeitsgruppe „Cooperation Issues“ tauscht sich beispielsweise zu generellen Policy-Fragen aus; die Arbeitsgruppen „Leniency“ (Kronzeugenprogramme) und „Ne bis in idem“ (Vermeidung doppelter Bestrafungen für dasselbe Vergehen) beraten über Fragen in den sie betreffenden Bereichen. In weiteren 15 sektoralen ECN-Untergruppen setzen sich die entsprechend spezialisierten Mitarbeiter der Behörden mit wettbewerbspolitischen Fragen in den verschiedenen Branchen bzw. Industrien auseinander. Die nur den ECN-Mitgliedern zugängliche Intranetplattform mit Namen „Circa“ ermöglicht es den Behördenmitarbeitern, sich via Internet über inhaltliche Themen auszutauschen und Arbeitsgruppen gemeinsam vorzubereiten.

Gerade in den Arbeitsgruppen werden Kontakte geknüpft und vertieft, die für den Arbeitsalltag der Wettbewerbsbehörden von Bedeutung sind. Die Arbeitsgruppen sind darüber hinaus ein Hinweis darauf, dass Deliberation und Lernen als Interaktionsmechanismen des Netzwerks etabliert werden sollen, um die Politik der Behörden zu koordinieren und eine einheitliche Wettbewerbskultur zu fördern. Diese böte die Chance, die Normbasierung des Netzwerks zu verstärken, sowie - aus der Sicht der GD Wettbewerb - dessen Fähigkeit zur Selbststeuerung zu verbessern und damit die Wahrscheinlichkeit einer hierarchischen Netzwerksteuerung zu verringern. Wie dies geschieht und was genau Akteure in den Mitgliedstaaten veranlasst, sich nach Zielen und Vorgaben der EU zu richten, bleibt jedoch ungeklärt (Benz 2007: 46). In der Literatur wurde immer wieder die Bedeutung der Institutionalisierung einer „Netzwerkkultur" hervorgehoben (Marsh/Smith 2000: 6).

Fraglich ist auch, wie es durch die Interaktionen der Wettbewerbsbehörden gelingen kann, die Resultate gemeinsamer Erörterungen in die politische Praxis der einzelnen Mitgliedstaaten zu übersetzen. Hier finden sich kaum überzeugende Konzepte bzw. Mechanismen. Dieses Problem wird dadurch ver- 
stärkt, dass wir in Gesprächen mit Behördenvertretern nur wenige Hinweise darauf finden konnten, dass in den Behörden selbst Prozesse in Gang gesetzt werden, mit denen die im ECN ausgetauschten Informationen und Ansätze hausintern abteilungsübergreifend verarbeitet würden.

Zwar verweisen Kassim und Wright (2009: 746) zu Recht darauf, dass das ECN weitgehend begrüßt wird und eine willkommene Austauschplattform bietet. Aber hinsichtlich der Schlussfolgerung, dies stärke die nationalen Wettbewerbsbehörden, nicht unbedingt aber die Kommission, ist zumindest Skepsis geboten. Der „Schatten der Hierarchie“, der sowohl in der rechtlichen Gestaltung als auch in den Köpfen der Netzwerkteilnehmer noch durchaus verankert ist, ist dann am wirkungsvollsten und gleichzeitig am wenigsten effizienzgefährdend für das Funktionieren des Netzwerks, wenn er keine Rolle in der Praxis des ECN spielt. Dies ist allerdings vor allem im technisch-organisatorischen Sinne nicht immer der Fall. Das ECN unterliegt durchaus gestalterischem Bemühen der Kommission, einem Network Management (Schout/ Jordan 2005: 210; Rhodes 2008). Gerber (2004: 13) spricht daher im Zusammenhang mit dem ECN sogar von einem „centralised interactive model“, auch weil die zentrale Position der Kommission durch die entsprechende Verordnung formal zementiert scheint. Und auch Cengiz (2007) argumentiert, dass es sich beim ECN um ein hierarchisches Netzwerk handelt, in dem unterschiedliche Standpunkte und Meinungen in weit geringerem Maße möglich sind als in einem vergleichbaren Netzwerk aus US-Staatsanwälten. „Zentralisiert“ ist vor allem der Netzwerkmodus, da das ECN wiederum als Plattform und Grundlage für das eigenständige Handeln der nationalen Wettbewerbsbehörden dienen soll. Eine eigene Abteilung innerhalb der GD Wettbewerb ist für das „Network Management" zuständig. Hier werden Treffen organisiert und die Intranetplattformen gepflegt und technisch überwacht. Es handelt sich beim ECN somit in erster Linie um ein Netzwerk, das nur in sehr geringem Maße selbst organisiert ist und das hinsichtlich seiner Abstimmungsverfahren stark formal geprägt ist, was abweichende Initiativen ressourcenstärkerer Wettbewerbsbehörden nicht ausschließt. Die Kommission initiiert in der Rolle eines zentralen Network Managers die Kommunikation im Netzwerk und hält sie in Gang. Als maßgebliche Organisatorin von Foren, Konferenzen und Arbeitsgruppen hat sie großen Einfluss auf die Agenda der wettbewerbspolitischen Deliberation im Netzwerk. Ihr Ziel ist, dass die einzelnen Netzwerkmitglieder die Position der Kommission kennen und danach handeln. In Interviews mit Mitarbeitern der Generaldirektion Wettbewerb ist immer wieder die Eigensicht als Hüterin der Verträge betont worden. Dafür zu sorgen, dass letztlich in ganz 
Europa nach einheitlichen Kriterien entschieden wird, wird als genuine Aufgabe der Kommission betrachtet. Das ist der Grund dafür, dass die Kommission sehr viel Wert darauf legt, Austausch über Zirkel und gemeinsame Abstimmungsrunden zu den relevanten Themen zu etablieren. Dass dies offenbar gelingt, erkennt man darin, dass 13 von 14 Behörden angegeben haben, sie würden die policy der Kommission kennen und sich danach richten. ${ }^{7}$ Dies wird weiter unten noch näher ausgeführt (vgl. Abschnitt c), „Effektivität“). Im Zuge der konkreten Fallbehandlung kontrolliert die Kommission darüber hinaus die avisierten Entscheidungen der einzelnen Behörden auf Kohärenz. Ihr kommt somit eine „monitoring role“ (Conseil de la Concurrence 2006: 63) innerhalb des Netzwerks zu. In der Praxis läuft dieses ,,monitoring“ zumeist konfliktfrei ab. Es wird relativ unpolitisch als betriebswirtschaftliche Optimierungsstrategie interpretiert und schmälert so in der Wahrnehmung der meisten Wettbewerbsbehörden nicht deren Entscheidungsautonomie.

Eine Vielzahl von Autoren (Scharpf 1993; Héritier 2002b; Börzel 2006a: 89 f.) argumentieren, dass auch neue Governance-Formen den „Schatten der Hierarchie" benötigen, um effektiv zu sein. Andere Autoren hingegen sehen gerade in der ,actor diversity“, also einer Struktur ohne zentralen Akteur, ein wichtiges Element eines funktionierenden Netzwerks (Wilks 2004: 68). Für das ECN ist es eine existentielle Frage, die Steuerungsambitionen der Kommission nicht überborden zu lassen. Einerseits ist die Kommission technisch (Umfang der Aufgabe) nicht mehr fähig, in einem Europa der 27 die Kartellkontrolle hierarchisch zu organisieren, andererseits verträgt die neue Rolle der nationalen Wettbewerbsbehörden als Akteure im Netzwerk bestenfalls eine auf Dauer gestellte organisatorische Hilfestellung der Kommission.

Für das ECN könnte sich aus einer machtvollen Stellung der Kommission zudem ein Problem ergeben, wenn traditionell tonangebende, weil ressourcenstarke Akteure wie das Bundeskartellamt oder das britische OFT sich bei Politik- oder Entscheidungsprozessen marginalisiert fühlen. Dann könnten sie sich offen oder versteckt gegen ausgehandelte Politikergebnisse wenden und so die Effizienz der Netzwerkkoordination gefährden. Konfliktpotential birgt außerdem die Tatsache, dass den nationalen Behörden eine routinemäßige Fallabwicklung zukommt, während die Kommission zum einen neue Kapazitäten freisetzen kann, um sich auf prestigeträchtige Fälle zu konzentrieren (und sie

7 Es ist nicht ganz auszuschließen, dass dieses Ergebnis auch eine Tendenz zu möglicherweise als „erwünscht“ vermuteten Antworten widerspiegelt. Dies würde jedoch die These eines „Schattens der Hierarchie“ weiter erhärten, denn „erwünscht“ ist dieses Ergebnis ja vor allem von der Kommission. 
auf diese Weise potentiell den nationalen Ämtern zu entziehen) und zum anderen Grundsatzentscheidungen und generelle Rechtsinterpretationen vornehmen kann.

Ein ungelöstes Steuerungsproblem für die Kommission ist der Umgang mit ressourcenstarken Netzwerkmitgliedern. Diese können selbstverständlich nicht die sich aus den rechtlichen Grundlagen des ECN ergebene Hierarchisierung des transgouvernementalen Netzwerkes aus den Angeln heben, haben sich aber eine eigenständige, zum Teil auch themensetzende Rolle im ECN gesichert. Eine Reihe von Erklärungen der Behörden deutet darauf hin, dass einzelne Ämter innerhalb des Netzwerks eine Art „kulturelle Hegemonie“ innehaben. Der französische Wettbewerbsrat „Conseil de la Concurrence“ schreibt in seinem Jahresbericht 2005 beispielsweise unverblümt: „The Conseil de la concurrence plays a major role in the ECN's activity“ (2006: 63). Das Bundeskartellamt nimmt eine ähnliche Rolle für sich in Anspruch. Zwar nehmen nach Angaben der Kommission auch kleinere Wettbewerbsbehörden in einigen Bereichen eine hervortretende Stellung ein, in der Gesamtsicht sind jedoch auch aus Sicht der Kommission die großen und gut ausgestatteten Behörden mit langer Tradition im ECN tonangebend. Unser Material hat zudem deutlich gezeigt, dass die großen Behörden das ECN zur Erweiterung ihrer Machtressourcen nutzen (können). Zum einen dient es dem Ausbau bzw. der Absicherung ihrer starken Stellung im Land. Das Bundeskartellamt kann jetzt beispielsweise europäisches Recht anwenden. Zum anderen gibt das ECN den großen nationalen Kartellämtern die Chance, über die Arbeit im Netzwerk den eigenen Einflussbereich auch auf andere Staaten der EU auszuweiten. Kleinere Wettbewerbsbehörden sind vor allem aus zwei Gründen am ECN interessiert. Erstens lernen sie von den erfahrenen Behörden und haben die Möglichkeit, Knowhow ins eigene Land zu transferieren; zweitens fehlt gerade den jüngeren Wettbewerbsbehörden eine kulturelle und politische Absicherung im eigenen Land. Mitarbeiter osteuropäischer Kartellämter klagten im Gespräch mit den Autoren beispielsweise über eine mangelnde Kooperationsbereitschaft der Polizei und mangelnde Akzeptanz von Seiten politischer Mandatsträger. Sechs von acht osteuropäischen Wettbewerbsbehörden, die an unserer Umfrage teilgenommen haben, kreuzten an, ihre Akzeptanz sei mittelmäßig. ${ }^{8}$ Je europäischer eine Entscheidung erscheint, desto größer ist in diesen Ländern die Chance auf poli-

8 Die Frage lautete: „In our country's politics the competition authorities enjoy...“. Ankreuzbar waren: very high importance, high importance, medium importance, low importance, not specified. 
tische Unterstützung für die eigene Arbeit. Hier dienen das ECN und der damit verbundene Verweis auf Kooperationen als Legitimationsquelle. Die Kommission hat letztlich das ECN geschaffen, um die eigene starke Stellung auszubauen. Alle beteiligten Akteure scheinen sich letztlich Machtgewinne zu versprechen. Ob dieses Win-Win-Szenario dauerhaft aufgeht, ist eine offene Frage. Das Konfliktpotential steigt, wenn die kleineren und jüngeren Wettbewerbsbehörden besser abgesichert, stärker und selbstbewusster werden - ein Trend, der sich derzeit abzuzeichnen scheint. Abzuwarten bleibt, wie die größeren Netzwerkteilnehmer, zu denen auch die Kommission gehört, reagieren, wenn sie ihre Deutungshoheit verlieren oder teilen müssen.

\section{c) Effektivität}

Eberlein und Newman nehmen an, dass in einem transgouvernementalen Netzwerk Ziele umso besser erreicht werden, je mehr Expertise die einzelnen Netzwerkbehörden in sich vereinen. Daneben identifizieren sie auch die regulatorische Autorität der im Netzwerk versammelten Behörden als Indikator für die Effektivität eines Netzwerks. Unter regulatorischer Autorität verstehen Eberlein und Newman beispielsweise die Möglichkeit, Verstöße zu rügen, Strafen zu verhängen oder Marktzugänge zu verweigern (2008: 36). Darauf wurde bereits bei der Darstellung der Befugnisse der Wettbewerbsbehörden (Tabelle 2) hingewiesen.

Unter Effektivität soll der Grad der Zielerreichung verstanden werden, unter Effizienz der Grad der Zielerreichung bei geringst möglichem Mitteleinsatz. Der von Eberlein und Newman indirekt Effektivität messende Indikator „Expertise" wird hier anhand zweier Indikatoren abgebildet: Erfahrungshorizont der nationalen Wettbewerbsbehörden und Bearbeitung von Fällen. Der erste Indikator ist der Zeitraum des Bestehens der ECN-Mitgliedsbehörden, also die Frage, seit wann es in den Mitgliedstaaten eine Wettbewerbsbehörde überhaupt gibt. Hier lassen sich deutliche Unterschiede feststellen. Verfügen die Europäische Union, Frankreich, Deutschland und Großbritannien ${ }^{9}$ über die erfahrensten Wettbewerbsämter, so haben die meisten anderen Mitgliedstaaten erst vor wenigen Jahren eine derartige Behörde ins Leben gerufen oder als unab-

9 Im britischen Wettbewerbs"regime" existiert neben dem Office of Fair Trading auch die Competition Commission, der das OFT wie auch die nationalen Regulierer Fälle übertragen können. Einschließlich ihrer Vorgänger ist die Competition Commission die älteste der europäischen Wettbewerbsbehörden (Gründungsdatum 1948). Sie wird in unseren Übersichten nicht erwähnt, weil sie kein offizielles Mitglied des ECNs ist. 
hängige Institution etabliert. In einigen Mitgliedstaaten, etwa in Griechenland, hatten zuvor politische Institutionen wie das Wirtschaftsministerium die Aufsicht über die Märkte inne. Die mittel- und osteuropäischen Länder hatten erst nach der Einführung der Marktwirtschaft infolge der Umwälzungen der Jahre 1989/90 überhaupt Bedarf an Wettbewerbsbehörden, die dort oftmals aus den einstigen Planungsbehörden hervorgingen.

Weiteren Aufschluss über die in einer Behörde vorhandene Expertise kann die Zahl der von dieser innerhalb eines bestimmten Zeitraums bearbeiteten Fälle geben. Tabelle 3 verdeutlicht die Unterschiede zwischen den Behörden. Dies geschieht jedoch lediglich annäherungsweise, da die Behörden sehr unterschiedlich berichten. Vor allem bei den Behörden der größeren Volkswirtschaften gibt es eine extrem hohe Zahl an Bagatellfällen, die meist auf dem „kleinem Dienstweg“ geklärt werden können, dennoch aber in die Statistik einfließen. Diese werden in den Jahresberichten der Behörden nicht immer separat ausgewiesen. Wo immer möglich wurden derartige Bagatellfälle herausgerechnet und die Zahl der tatsächlich mit einer Entscheidung abgeschlossenen Fälle angegeben. 
Tabelle 3: Abgeschlossene Fälle der ECN-Mitglieder

\begin{tabular}{|c|c|c|c|c|c|c|c|c|c|}
\hline & \multicolumn{4}{|c|}{$\begin{array}{l}\text { abgeschlossene Fälle Kartelle und } \\
\text { Missbrauch }\end{array}$} & \multicolumn{4}{|c|}{ abgeschlossene Fälle Fusionen } & \multirow[t]{2}{*}{ Summe } \\
\hline & 2004 & 2005 & 2006 & Gesamt & 2004 & 2005 & 2006 & Gesamt & \\
\hline$\overline{A U T}$ & 175 & 40 & 104 & 319 & 608 & 333 & 722 & 1663 & 1982 \\
\hline$\overline{B E L}$ & k.A. & k.A. & k.A. & k.A. & k.A. & k.A. & k.A. & k.A. & k.A. \\
\hline$B U L$ & 63 & 58 & 33 & 154 & 24 & 39 & 60 & 123 & 277 \\
\hline$\overline{C Y P}$ & 12 & 33 & k.A. & 45 & 5 & 4 & k.A. & 9 & 54 \\
\hline$C Z E$ & 29 & 9 & 8 & 46 & 134 & 55 & 61 & 250 & 296 \\
\hline$D A N$ & 12 & 16 & 15 & 43 & 12 & 11 & 8 & 31 & 74 \\
\hline$E S T$ & 49 & 35 & 23 & 107 & 38 & 41 & 35 & 114 & 221 \\
\hline$E S P$ & 91 & 86 & 92 & 269 & 94 & 115 & 132 & 341 & 610 \\
\hline$\overline{E U}$ & 28 & 41 & 45 & 114 & 247 & 310 & 356 & 913 & 1027 \\
\hline$F I N$ & 46 & 31 & 46 & 123 & 90 & 55 & 62 & 207 & 330 \\
\hline$F R A$ & 100 & 105 & 103 & 308 & 130 & 118 & 128 & 376 & 684 \\
\hline GER & 135 & 164 & 135 & 434 & 1412 & 1687 & 1829 & 4928 & 5362 \\
\hline$\overline{G R E}$ & 7 & 7 & 16 & 30 & 10 & 14 & 13 & 37 & 67 \\
\hline HUN & 56 & 45 & 50 & 151 & 65 & 70 & 43 & 178 & 329 \\
\hline IRL & 25 & 61 & 72 & 158 & 94 & 95 & 105 & 294 & 452 \\
\hline$I T A$ & 84 & 18 & 21 & 123 & 612 & 596 & 717 & 1925 & 2048 \\
\hline$L A T$ & 45 & 35 & 37 & 117 & 9 & 17 & 28 & 54 & 171 \\
\hline$L I T$ & 79 & 81 & 85 & 245 & 56 & 64 & 61 & 181 & 426 \\
\hline$L U X$ & k.A. & k.A. & k.A. & $k . A$. & k.A. & k.A. & k.A. & $k . A$. & k.A. \\
\hline$\overline{M A L}$ & k.A. & k.A. & k.A. & $k . A$. & k.A. & k.A. & k.A. & $k . A$. & k.A. \\
\hline NED & 22 & 20 & 26 & 68 & 83 & 80 & 135 & 298 & 366 \\
\hline POL & 152 & 144 & 202 & 498 & 218 & 329 & 263 & 810 & 1308 \\
\hline POR & 22 & 34 & 34 & 90 & 76 & 67 & 73 & 216 & 306 \\
\hline$R O M$ & 77 & 46 & 34 & 157 & 165 & 115 & 120 & 400 & 557 \\
\hline$S V K$ & 42 & 30 & 32 & 104 & 79 & 69 & 54 & 202 & 306 \\
\hline$S L O$ & k.A. & 5 & 5 & 10 & k.A. & 54 & 46 & 100 & 110 \\
\hline$S W E$ & 115 & 122 & 147 & 384 & 268 & 305 & 175 & 748 & 1132 \\
\hline$\overline{U K}$ & 48 & 36 & 23 & 107 & 257 & 242 & 131 & 630 & 737 \\
\hline
\end{tabular}

Die Jahresberichte der Behörden Belgiens, Luxemburg und Maltas waren leider nicht verfügbar.

Quelle: Eigene Zusammenstellung nach den Jahresberichten der Behörden. 
Als besonders erfahren können jene Behörden gelten, die sehr lange existieren und zugleich hohe Fallzahlen verbuchen. Dies gilt für die Europäische Kommission, die beiden französischen Behörden, das Bundeskartellamt und das britische OFT. Österreich, Italien, Polen, Schweden und Spanien verfügen zwar ebenfalls über Behörden, die eine hohe Zahl von Fällen bearbeitet haben, diese Wettbewerbsbehörden haben aber eine deutlich kürzere Geschichte. Wenig überraschend finden sich die Behörden Zyperns, Estlands oder Lettlands eher am Ende dieser Skala. Im Kontext der Analyse des ECN bestätigt dieser Befund die schon oben getroffene Feststellung einer asymmetrischen Expertise der nationalen Wettbewerbsbehörden.

Die ausschließliche Konzentration auf Erfahrung reicht als analytischer Zugang zu transgouvernementalen Netzwerken nicht aus. Große Erfahrung der Behörden wird hier nach Eberlein und Newman als Indiz dafür betrachtet, dass diese im Netzwerk die ihnen gesetzten Ziele erreichen. Entscheidend ist jedoch auch, wie diese Ziele erreicht werden. Neben der Effektivität ist also die Effizienz von Belang. Zentral ist die Frage, ob bei der Umsetzung der inhaltlichen Ziele im Netzwerk Reibungsverluste entstehen, die mit hohen Transaktionskosten einhergehen. Ein eigener Strang der politikwissenschaftlichen Netzwerkforschung beschäftigt sich mit der Frage, wie Netzwerke zu managen sind. Wie Rhodes (2008: 433) zeigt, geht hier der institutionelle Ansatz davon aus, dass Netzwerke in Richtung einer Veränderung von Anreizstrukturen, Regeln und Kulturen wirken können. Genau dies ist der Zweck des ECNs.

Angenommen wird, dass Network Governance in konkreten Entscheidungssituationen die Effektivität steigert, ,weil dauerhafte Interaktionen den strategischen Einsatz von Verhandlungsmacht ausschließen, der zur Konflikteskalation und zum Scheitern einer Einigung führen kann“ (Benz 2007: 41). In jedem Netzwerk kooperieren die teilnehmenden Akteure jedoch, weil sie bestimmte persönliche und organisationale Ziele erreichen wollen. Jeder einzelne Akteur verfolgt eigene Interessen. Hier verweist die politikwissenschaftliche Netzwerkforschung darauf, dass die modernen Gesellschaften an Komplexität erheblich zugenommen haben. Keine Organisation ist in der Lage, die eigenen Ziele und Interessen alleine zu erreichen bzw. durchzusetzen, sondern ist dafür auf die Unterstützung anderer angewiesen. Es entsteht eine Ressourcenabhängigkeit von anderen Akteuren (Rhodes 1996: 658).

Dies ist im Hinblick auf die Steuerung im ECN von höchster Relevanz. Die Interdependenz der Ressourcennutzung zwischen den einzelnen Wettbewerbsbehörden kann ein wichtiger Beitrag zur Relativierung der Möglichkeiten hierarchisierten Entscheidens im ECN werden. Fest steht, dass diese Interdepen- 
denz wächst. Einer der Gründe hierfür ist die ökonomische Integration in der Europäischen Union. Im Zuge der europa- und weltweit zunehmenden Unternehmenszusammenschlüsse meist transnationalen Charakters sind einzelstaatliche Kartellbehörden oft nicht mehr in der Lage, alleine Verstöße gegen das Wettbewerbsrecht zu ahnden. Gegenseitige Unterstützung, beispielsweise bei der Ermittlung von Sachverhalten, und der gegenseitige Austausch von Informationen sind daher im Sinne aller Behörden. Das schließt im ECN auch den lange geforderten Austausch vertraulicher Informationen ein. Zudem führt die Tatsache, dass ökonomische Transaktionen in zunehmendem Maße gleichzeitig mehrere EU-Mitgliedstaaten betreffen, dazu, dass das europäische Recht immer häufiger angewendet wird und in der Folge die Bedeutung der nationalen Kartellrechtsregelungen abnimmt (Budzinski 2006). Die Kommission kann darauf setzen, dass eine Zunahme der Anwendung europäischen Rechts stärkere Legitimität erfährt, wenn dies durch nationale Behörden geschieht.

Tabelle 4: Neue Fälle im Zeitraum 1. Mai 2004 bis 30. Juni 2008, über die das ECN durch eine Wettbewerbsbehörde informiert wurde

\begin{tabular}{|l|l|l|}
\hline Mitgliedstaat & $\begin{array}{l}\text { Neue Fälle, bei denen das ECN } \\
\text { informiert wurde }\end{array}$ & $\begin{array}{l}\text { Anteil an der Gesamtzahl neuer Fälle, } \\
\text { bei denen das ECN informiert wurde }\end{array}$ \\
\hline AUT & 18 & $2,02 \%$ \\
\hline BEL & 32 & $3,59 \%$ \\
\hline BUL & 8 & $0,90 \%$ \\
\hline CYP & k.A. & k.A. \\
\hline CZE & 10 & $1,12 \%$ \\
\hline DAN & 48 & $5,38 \%$ \\
\hline ESP & 41 & $4,60 \%$ \\
\hline EST & 6 & $0,67 \%$ \\
\hline EU & 159 & $17,83 \%$ \\
\hline FIN & 15 & $1,68 \%$ \\
\hline FRA & 145 & $16,26 \%$ \\
\hline GER & 91 & $10,20 \%$ \\
\hline GRE & 19 & $2,13 \%$ \\
\hline HUN & 60 & $6,73 \%$ \\
\hline IRL & 9 & $1,01 \%$ \\
\hline ITA & 36 & $4,04 \%$ \\
\hline LAT & 5 & $0,56 \%$ \\
\hline LIT & 3 & $0,34 \%$ \\
\hline LUX & 1 & $0,11 \%$ \\
\hline MAL & 1 & $0,11 \%$ \\
\hline & &
\end{tabular}


Europäisches Regieren in der Kartellkontrolle

\begin{tabular}{|l|l|l|}
\hline NED & 60 & $6,73 \%$ \\
\hline POL & 18 & $2,02 \%$ \\
\hline POR & 21 & $2,35 \%$ \\
\hline ROM & 1 & $0,11 \%$ \\
\hline SVK & 7 & $0,78 \%$ \\
\hline SLO & 10 & $1,12 \%$ \\
\hline SWE & 27 & $3,03 \%$ \\
\hline UK & 41 & $4,60 \%$ \\
\hline Total & $\mathbf{8 9 2}$ & $\mathbf{1 0 0 , 0 0} \%$ \\
\hline
\end{tabular}

Quelle: http://ec.europa.eu/comm/competition/ecn/statistics.html

Interdependenz ist nicht nur eine formale Kategorie. Sie beruht auf und schafft eine bestimmte Dichte der Kommunikation (Tabelle 4) und ein hohes Maß an Vertrauen unter den Mitgliedern (Hajer/Versteeg 2005: 342; Wake Carroll/Carroll 1999: 19). Denn in allen Fällen ist die Schwelle überschritten, nach der ein Fall nach europäischem Recht zu behandeln ist. Das heißt auch, dass in aller Regel ein Austausch zwischen mindestens zwei nationalen Wettbewerbsbehörden stattfindet und Informationen ausgetauscht werden. Die Kommission ist ebenfalls automatisch involviert, betont aber im Interview vor allem die informellen Mechanismen neben den formalen Benachrichtigungen. Anzumerken ist, dass diese auf formale Verfahren gestützt sind, weil ohne die internetbasierte Anwendung kein Austausch zustande gekommen wäre. Es komme dann aber häufig vor, dass Mitarbeiter der Behörden anriefen und nach Einschätzungen und Erfahrungen fragten. Vertrauen und Deliberation sind Garanten für einen möglichen Verzicht auf hierarchische Steuerungsbemühungen.

Als problematisch könnte sich die Größe des Netzwerks erweisen. Für Benington und Harvey (1996: 163) sind interne Transparenz, Demokratie und Offenheit beim Policy-Making entscheidende Voraussetzungen für ein „,funktionierendes Netzwerk". Die Netzwerkgröße ist somit im Hinblick auf die Funktionsfähigkeit limitiert. Am ECN partizipieren 30 nationale Wettbewerbsbehörden und die Kommission, jede dieser Institutionen mit eigenem Mitarbeiterstab. Gegenseitiger Austausch findet in den Arbeitsgruppen und Meetings statt, hier werden persönliche Kontakte geknüpft. Aber einen detaillierten Überblick über die Aktivitäten und Teilnehmer des ECNs haben allenfalls die Mitarbeiter des Chef-Koordinators für das ECN in Brüssel. Somit ist zumindest fraglich, ob die Begriffe Transparenz, Demokratie und Offenheit nach innen auf das ECN anwendbar sind. 
Von den Mitgliedern des ECN wird dieses jedoch eher als hilfreich empfunden. Immer wieder wird auf die Schaffung und Vertiefung informeller Kontakte verwiesen, die sich bei der täglichen Arbeit auszahlen würden. Diese Einschätzung dürfte auch damit zusammenhängen, dass die Kommission von ihren hierarchischen Instrumenten zur Entscheidungssteuerung, insbesondere im Hinblick auf das Tagesgeschäft der Fallallokation, bislang keinen Gebrauch gemacht hat. Bis Mai 2009 haben die nationalen Wettbewerbsbehörden mehr als 300 Fälle gemeldet. Die Kommission berichtet, sie habe in den rund fünf Jahren, in denen die Verordnung in Kraft ist, kein einziges Mal ein Verfahren nach Artikel 11 Absatz 6 eingeleitet, um die Zuständigkeit an sich zu ziehen. Dies wird im Zusammenhang mit dem informellen Austausch gesehen, der auch von den nationalen Wettbewerbsbehörden betont wird (Europäische Kommission 2009: 8-9). Hinweise auf die Frage, warum die Kommission nicht eingreifen muss, liefern auch die Befragungsdaten.

Tabelle 5: Einfluss der Kommission aus Sicht der Wettbewerbsbehörden

\begin{tabular}{|l|c|}
\hline Frage & Antworthäufigkeiten* \\
\hline Commission merely provides know-how & 2 \\
\hline Commission points out its position on content & 2 \\
\hline Sets requirements and directives & 2 \\
\hline $\begin{array}{l}\text { Often, our authorities request the position of } \\
\text { the Commission at an early stage of decision- } \\
\text { making, i.e. before the information duty of Ar- } \\
\text { ticle } 11(4) \text { Regulation } 1 / 2003 \text { comes into effect }\end{array}$ & 13 \\
\hline $\begin{array}{l}\text { We know the policy of the Commission and act } \\
\text { on that policy }\end{array}$ & 3 \\
\hline $\begin{array}{l}\text { In the decision-making process, we have to } \\
\text { keep in mind that the Commission can retrieve } \\
\text { a case if we take a decision that is not in line } \\
\text { with Commission's policy }\end{array}$ \\
\hline $\mathrm{N}=14$ \\
\hline
\end{tabular}

* Bis zu drei Kreuze waren möglich.

Tabelle 5 liefert Hinweise darauf, dass die Wettbewerbsbehörden gewillt sind, der Policy der Kommission zu entsprechen. Aus Sicht der Behörden ist es der Kommission gelungen, die eigene Position ausreichend klar zu machen. Es lassen sich überdies keine Indizien dafür finden, dass Widersprüche und Klagen im Zuge der Neuregelung zugenommen hätten. Einigkeit besteht unter allen Beteiligten darin, dass der Schwerpunkt der Netzwerkaktivität auf Informa- 
lität liegt. Und solange dies so ist, scheint die Zustimmung der beteiligten nationalen Ämter jedenfalls sicher. Bislang scheint die Fallallokation nur in wenigen Fällen zu Konflikten geführt zu haben.

\section{Transgouvernementales Regieren in der Kartellkontrolle-Schritte zur Enthierarchisierung eines transgouvernementalen Netzwerks?}

Die Europäische Union hat sich mit der Reform der Kartellkontrolle 2004 ein, wie gezeigt werden konnte, relativ flexibles Instrument gegeben. Dieses kann als hierarchisiertes transgouvernementales Netzwerk beschrieben werden, das allerdings neben den von Eberlein und Newman genannten Charakteristika noch eine Reihe weiterer Merkmale aufweist. Insbesondere aus der Perspektive politischer Steuerung sind die bisherigen Analyseansätze zum Verständnis transgouvernementaler Netzwerke weiterzuentwickeln. Es stellt sich heraus, dass die Konstruktion des ECN, wie Rational-Choice- und Resource-Dependency-Ansätze erwarten ließen (Rhodes 2008: 431), nicht nur als Koordinationsinstrument der Arbeit nationaler Kontrollbehörden zu verstehen ist. Ebenso wenig genügt der Blick in die Rechtsgrundlagen des ECN, der sehr rasch und eindringlich auf die Hierarchisierung des ECN aufmerksam macht. Selbststeuerung des ECN ist aus der Sicht der Kommission ebenso ausgeschlossen, wie dies die Re-Hierarchisierung des ECN aus der Sicht der Wettbewerbsbehörden ist.

Das Prinzipal-Agent-Problem ist zwar per se nach den Buchstaben des Gesetzes geklärt. De facto stellt es aber vor allem deshalb kein Funktionshindernis für das ECN dar, weil die Kommission ihre Führungsrolle auf die Unterstützung der organisatorischen Plattform des ECN reduziert und mit Blick auf den festgestellten „Schatten der Hierarchie“ auch reduzieren kann. Zugleich sieht sie zu ihrer Zufriedenheit, dass in immer größerem Maße europäisches Wettbewerbsrecht und zum Teil auch die europäische Wettbewerbsphilosophie des „more economic approach“10 in nationale Gesetzgebung übernommen wird. Informalität, Kommunikation und eine gewisse Zurückhaltung der Kommission prägen den Alltag im ECN. Arbeitsgruppen zur Problemlösung erwecken den Eindruck der symmetrischen Beteiligung aller. Die Hierarchisierung des transgouvernementalen Netzwerkes wird vor allem dann noch deutlich, wenn die EU als agenda setter in der Kartellkontrolle auftritt.

10 Ausführlich zum Konzept des „,more economic approach“ siehe z.B. Steinke 2011.

ZPol 3-4/10 
Trotz aller Harmoniebekundungen, mit der die Realität des ECN v. a. nach außen präsentiert wird, bleibt es dabei, dass das Steuerungsdilemma für die Kommission lautet: Weiterer Hierarchieverlust oder geringere Netzwerkeffizienz. Der bisher entstandene Status Quo der Netzwerkentscheidungen verdeckt zwei nichtintendierte Konsequenzen der Netzwerksteuerung, die oben herausgearbeitet wurden: (1) Die Netzwerkasymmetrien, die sich aus der unterschiedlichen Ressourcenausstattung im breitesten Sinne zwischen einzelnen Wettbewerbsbehörden ergeben. Diese können im Konfliktfall auch zum Nukleus von Gruppen „gleichgesinnter" Wettbewerbsbehörden werden. Solchen Gruppenbildungen hat die Kommission durch ihre besonders hilfreiche Haltung gegenüber „kleinen“ Behörden bisher erfolgreich gegengesteuert. Netzwerkasymmetrien sind aber nicht zu vermeiden. Allen Mitgliedern des Netzwerks sind die machtpolitischen Knotenpunkte bzw. die unterschiedlichen wettbewerbspolitischen Präferenzen der ressourcenstarken Wettbewerbsbehörden bekannt. (2) Die wachsende Autonomie der nationalen Wettbewerbsbehörden bei der alltäglichen Aufgabe der Fallbearbeitung. Selbstverständlich kommen die nationalen Wettbewerbsbehörden weiterhin ihren Informationspflichten gegenüber der Kommission nach. Diese könnte allerdings durchaus in der Zukunft davon überrascht werden, dass wenn sie nach langer Zeit einmal aus übergeordneten Gründen wieder hierarchisch Entscheidungen des ECN steuern möchte, sie auf Unverständnis bei den betroffenen Wettbewerbsbehörden stößt, die nun gewohnt sind im gegenseitigen Austausch grenzüberschreitende Fälle zu behandeln. Dieses Szenario ist durchaus nicht unrealistisch, bedenkt man, dass Vertreter der Wirtschaft und Anwälte im Rahmen der Bewertung der Verordnung 1/2003 kritisiert haben, dass es unterschiedliche Standards bei der Bewertung bestimmter Verhaltensweisen gibt - also letztlich von den Wettbewerbsbehörden selbst so nicht angezeigte Inkonsistenzen, die für die Kommission im Grunde nicht hinnehmbar sind (Europäische Kommission 2009: 7). Selbstverständlich kann die Kommission auch die Gegenstrategie forcieren und noch mehr als dies bisher gelegentlich der Fall war, darauf pochen, dass die ,wichtigsten“ Fälle in Brüssel landen. Das wäre allerdings der erste Schritt zur Reduzierung des ECN von einem ,governance"-Versuch zu einem formalen interorganisatorischen Netzwerk mit Kollateralschäden bei der Legitimation und Implementation europäischer Kartellkontrollpolitik. Die Befürchtung, dass die Kommission eine solche Strategie wählen könnte, ist gerade vor dem Hintergrund der immer noch eher schwierigen konjunkturellen Lage im europäischen Binnenmarkt hoch. Im Interview erklärte ein namhafter und einflussreicher New Yorker Wettbewerbsrechtsexperte gegenüber den Verfassern dieses Artikels, dass gerade 
kleinere nationale Wettbewerbsbehörden sich im Zuge der Wirtschafts- und Finanzkrise sorgten, dass die Kommission mehr Fälle an sich ziehen könnte. Europäisches Regieren in der Kartellpolitik stellt sich also derzeit dar als prekäres und zum Teil auch fragiles Gleichgewicht nichtintendierter, durch Netzwerkasymmetrien und Autonomiebestrebungen nationaler Wettbewerbsbehörden erzeugte Fliehkräfte und auf formalisierte Verfahren gestützte Kooperationsstrategien in einem transgouvernementalen Netzwerk.

\section{Literatur}

Benington, John/Harvey, Janet, 1996: Transnational local authority networking within the European Union: passing fashion or new paradigm?, in: G. Bruce Doern/Stephen Wilks (Hrsg.), Comparative Competition Policy. National Institutions in a Global Market, Oxford, 149-166.

Benz, Arthur, 2007: Entwicklung von Governance im Mehrebenensystem der EU, in: Ingeborg Tömmel (Hrsg.), Die Europäische Union: Governance und Policy-Making, Wiesbaden, 34-55.

Börzel, Tanja A., 2006a: Coping with Accession. New Modes and EU Enlargement, in: Gunnar Folke Schuppert (Hrsg.), The Europeanisation of Governance, Baden-Baden, 77-92.

Börzel, Tanja A., 2006b: European Governance - nicht neu, aber anders, in: Schuppert, Gunnar Folke (Hrsg.), Governance-Forschung. Vergewisserung über Stand und Entwicklungslinien, Baden-Baden, 72-94 (2. Aufl.).

Börzel, Tanja A., 2007: European Governance - Verhandlungen und Wettbewerb im Schatten der Hierarchie, in: Ingeborg Tömmel (Hrsg.), Die Europäische Union: Governance und Policy-Making, Wiesbaden, 59-89.

Budzinski, Oliver, 2006: Modernisierung der europäischen Wettbewerbsordnung: Werden die nationalen Wettbewerbspolitiken verdrängt?, Marburg Working Papers on Economics Nr. 200608, Marburg.

Cengiz, Firat, 2007: Managing Networks Between the Competition Authorities in the EC and the US: Different Polities, Different Designs, in: European Competition Journal 3 (2), 413-436.

Cini, Michell/McGowan, Lee, 1998: Competition Policy in the European Union, London.

Conseil de la Concurrence, 2006: 2005 Activity Report. Paris, http://www. conseil-concurrence.fr/doc/rapport05_ang.pdf (Stand: 15.12.2010). 
Damgard, Bodil, 2006: Do Policy Networks lead to Network Governing ?, in: Public Administration 84 (3), 673-691.

Dowding, Keith, 1995: Model or Metaphor? A Critical Review of the Policy Network Approach, in: Political Studies 63 (1), 136-158.

Eberlein, Burkard/Kerwer, Dieter, 2002: Theorizing the New Modes of European Union Governance, in: European Integration Online Papers 5, http:// eiop.or.at/eiop/texte/2002-005.htm (Stand: 15.12.2010).

Eberlein, Burkard/Kerwer, Dieter, 2004: New Governance in the European Union. A Theoretical Perspective, in: Journal of Common Market Studies 42 (1), 121-142.

Eberlein, Burkard/Newman, Abraham L., 2008: Escaping the International Governance Dilemma? Incorporated Transgovernmental Networks in the European Union, in: Governance 21 (1), 25-52.

Europäische Kommission, 2004a: Eine proaktive Wettbewerbspolitik für ein wettbewerbsfähiges Europa. Mitteilung der Kommission, Brüssel (KOM(2004) 293 endgültig).

Europäische Kommission, 2004b: Bekanntmachung der Kommission über die Zusammenarbeit innerhalb des Netzes der Wettbewerbsbehörden, Amtsblatt der Europäischen Union, 2004 C 101/03, 43-53.

Europäische Kommission, 2009: Bericht über das Funktionieren der Verordnung (EG) Nr. 1/2003 des Rates, Brüssel \{SEK(2009)574\}.

Falkner, Gerda/Treib, Oliver/Hartlapp, Miriam/Leiber, Simone, 2005: Complying with Europe. EU Harmonisation and Soft Law in the Member States, Cambridge u. a.

Gerber, David, 2004: The Evolution of a European Competition Law Network, in: Claus-Dieter Ehlermann/Isabela Atanasiu (Hrsg.), European Competition Law Annual 2002: Constructing the EU Network of Competition Authorities, Oxford u. a., 12-14.

Gerber, David, 2008: Two Forms of Modernization in European Competition Law, in: Fordham International Law Journal 31 (5), 1235-1265.

Global Competition Review, 2008a: The Handbook of Competition Enforcement Agencies 2008, London.

Global Competition Review, 2008b: The European Antitrust Review 2008, London.

Hajer, Maarten/Versteeg, Wytske, 2005: Performing Governance through Networks, in: European Political Science 4 (3), 340-347. 
Héritier, Adrienne, 2002a: New Modes of Governance in Europe: PolicyMaking Without Legislating? (Reihe Politikwissenschaft, 81), http://www. ihs.ac.at/publications/pol/pw_81.pdf (Stand: 15.02.2011).

Héritier, Adrienne (Hrsg.), 2002b: Common Goods. Reinventing European and International Governance, Lanham/Oxford.

Kassim, Hussein/Wright, Kathryn, 2009: Bringing Regulatory Process Back In: The Reform of EU Antitrust and Merger Control, in: West European Politics 32 (4), 738-755.

Knill, Christoph/Lenschow, Andrea, 2003: Modes of Regulation in the Governance of the European Union: Towards a Comprehensive Evaluation, in: European Integration Online Papers 7, http://eiop.or.at/eiop/texte/2003001a.htm (Stand: 15.12.2010).

Kohler-Koch, Beate, 1999: The Evolution and Transformation of European Governance, in: Rainer Eising/Beate Kohler-Koch (Hrsg.), The Transformation of Governance in the European Union, London/New York, 14-35.

Marsh, David/Smith, Martin, 2000: Understanding Policy Networks: towards a Dialectical Approach, in: Political Studies 48 (1), 4-21.

Marsh, David/Smith, Martin, 2001: There is More than One Way to Do Political Science: On Different Ways to Study Policy Networks, in: Political Studies 49 (3), 526-541.

McGowan, Lee, 2005: Europeanization unleashed and rebounding: assessing the modernization of EU cartel policy, in: Journal of European Public Policy 12 (6), 986-1004.

McGowan, Lee, 2007: Theorizing European integration: Revisting neofunctionalism and testing its suitability for explaining the development of EC competition policy? in: European Integration Online Papers 3, http://eiop. or.at/eiop/index.php/eiop/article/view/2007_003a/50 (Stand: 15.12.2010).

Müller, Markus M./Sturm, Roland, 2010, Wirtschaftspolitik kompakt, Wiesbaden.

Niskanen, William A., 1971: Bureaucracy and Representative Government, Chicago.

Peters, B. Guy, 2010: The Politics of Bureaucracy. An Introduction to Comparative Public Administration, London (6. Aufl.).

Powell, Walter W., 1990: Neither Market nor Hierarchy: Network Forms of Organization, in: Research in Organizational Behavior 12, 295-336.

Rhodes, R.A.W., 1996: The New Governance: Governing without Government, in: Political Studies 44 (4), 652-667. 
Rhodes, R.A.W., 2000: Governance and public administration, in: Jon Pierre (Hrsg.), Debating Governance: Authority, steering and democracy, Oxford, 54-90.

Rhodes, R.A.W., 2008: Policy Network Analysis, in: Michael Moran/Martin Rein/Robert E. Goodin (Hrsg.), The Oxford Handbook of Public Policy, New York, 425-447.

Riley, Alan, 2003: EC Antitrust Modernisation: The Commission Does Very Nicely - Thank You! Part One: Regulation 1 and the Notification Burden, in: European Competition Law Review 24 (11), 604-615.

Scharpf, Fritz W., 1993: Positive und negative Koordination in Verhandlungssystemen, in: Adrienne Héritier (Hrsg.), Policy Analyse. Kritik und Neuorientierung, Opladen, 57-83.

Schmidt, Susanne K./Blauberger, Michael/van den Nouland, Wendelmoet, 2007: Jenseits von Implementierung und Compliance - Die Europäisierung der Mitgliedstaaten, in: Ingeborg Tömmel (Hrsg.), Die Europäische Union: Governance und Policy-Making, Wiesbaden, 273-294.

Schmidt, Ingo/Schmidt, André, 2006: Europäische Wettbewerbspolitik und Beihilfenkontrolle, München.

Schout, Adriaan/Jordan, Andrew, 2005: Coordinated European Governance: Self-Organizing or Centrally Steered?, in: Public Administration 83 (1), 201-220.

Steinke, Joß, 2011: „Leaving the Cowboy Hat at Home?” Die Neuausrichtung der europäischen Wettbewerbspolitik als Politiktransfer aus den USA. Im Erscheinen.

Sturm, Roland/Zimmermann-Steinhart, Petra, 2003: Das Europa des freien Marktes. Funktion und Konsequenzen der europäischen Wettbewerbspolitik, in: Gesellschaft-Wirtschaft-Politik 52 (3), 383-409.

Tömmel, Ingeborg, 2007: Governance und Policy-Making im Mehrebenensystem der EU, in: Ingeborg Tömmel (Hrsg.): Die Europäische Union: Governance und Policy-Making, Wiesbaden, 11-33.

van Waarden, Frans/Drahos, Michaela, 2002: Courts and (Epistemic) Communities in the Convergence of Competition Policies, in: Journal of European Public Policy 9 (6), 913-934.

Wake Carroll, Barbara/Carroll, Terrance, 1999: Civic Networks, Legitimacy and the Policy Process, in: Governance 12 (1), 1-28.

Wilks, Stephen, 2004: Understanding Competition Policy Networks in Europe: A Political Science Perspective, in: Claus-Dieter Ehlermann/Isabela Atana- 
siu (Hrsg.), European Competition Law Annual 2002: Constructing the EU Network of Competition Authorities, Oxford u. a., 65-79.

Wilks, Stephen, 2005: Agency Escape: Decentralization or Dominance of the European Commission in the Modernization of Competition Policy?, in: Governance 18 (3), 431-452.

Wilks, Stephen, 2007: Agencies, Networks, Discourses and the Trajectory of the European Competition Enforcement, in: European Competition Journal 3 (2), 437-464.

Korrespondenzanschrift:

Dipl.-Pol. Ingo Schorlemmer

Rheinvillenstraße 4

68163 Mannheim

Tel: +49(0)621/16769657

E-Mail: ingo_schorlemmer@web.de

Dipl.-Pol. Joß Steinke

Institut für Arbeitsmarkt- und Berufsforschung

der Bundesagentur für Arbeit

Weddigenstraße 20-22

90478 Nürnberg

Tel: +49(0)911/179-2189

Fax: $+49(0) 911 / 179-3380$

E-Mail: joss.steinke@iab.de

Professor Dr. Roland Sturm

Institut für Politische Wissenschaft

Friedrich-Alexander-Universität Erlangen-Nürnberg

Kochstraße 4

91054 Erlangen

Tel: +49(0)9131/85-22370

Fax: 49(0)9131/85-22371

E-Mail: rdsturm@phil.uni-erlangen.de 